\title{
Spin-orbit induced noncubic charge distribution in cubic ferromagnets. II. Tight-binding analysis
}

\author{
G. Seewald and E. Zech \\ Physik-Department, Technische Universität München, D-85748 Garching, Germany \\ H. Haas \\ Bereich Strukturforschung, Hahn-Meitner-Institut Berlin, D-14109 Berlin, Germany \\ and EP Division, CERN, CH-1211 Geneva 23, Switzerland
}

(Received 6 April 2002; published 4 November 2002)

\begin{abstract}
The orbital moment and the noncubic charge distribution in ferromagnetic transition metals with cubic lattice symmetry are investigated within the tight-binding model. By combining the tight-binding approximation, perturbation theory, and the Green's function formalism for impurity scattering, approximate expressions for both effects are derived that depend only on the spin-orbit coupling strength and the density of states of the system without spin-orbit coupling. The basic relations between the orbital moment, the noncubic charge distribution, and the band structure are derived from the form of these expressions and from their application to various model band structures: We explain in this way the scaling with the spin-orbit coupling strength and bandwidth, the typical order of magnitude, the variation as a function of the band filling, the sensitivity to band structure details, and the role of the splitting between spin-up and spin-down states. For the noncubic charge distribution we derive the form of the dependence on the direction of the magnetization and show how the sign and magnitude of this anisotropy are related to the different energy distributions of $e_{g}$ and $t_{2 g}$ states. This tight-binding analysis is finally applied to the $5 d$ impurities in Fe. The local densities of states without spin-orbit coupling are obtained by self-consistent augmented plane-wave calculations using a supercell method. The special features of the $5 d$ impurities in Fe with respect to the band structure, the orbital moment, and the noncubic charge distribution are discussed. The general trend of the systematics is interpreted as a band filling effect. The prevailing sign of the anisotropy is ascribed to the concentration of the $e_{g}$ states near the Fermi energy. The results of the tight-binding analysis are compared with the experiment and a more rigorous calculation.
\end{abstract}

DOI: $10.1103 /$ PhysRevB.66.174402

PACS number(s): 75.50.Bb, 71.20.Be

\section{INTRODUCTION}

The spin-orbit coupling (SOC) is only a small contribution to the energy of the valence electrons in transition metals if compared with the bandwidth or the exchange interaction. But it plays a key role for several important phenomena in the magnetism of Fe-, Co-, or Ni-based compounds, like the magneto-optic Kerr effect, magnetic anisotropy energy, or magnetostriction. The calculation of these spin-orbit effects from first principles and their properties in artificially structured material have found much interest in recent years. ${ }^{1-6}$ In this work we investigate a spin-orbit effect that was less intensely studied in the past: the noncubic charge distribution in ferromagnets with cubic lattice symmetry. It can be measured via the electric field gradient (EFG) at the nuclear site and is a sensitive test for the theory of spin-orbit effects in transition metals.

For a long time data were available only for a few favorable cases. But due to recent improvements in the measurement technique, a more complete experimental study of the spin-orbit induced EFG (SO-EFG) has become feasible. A first systematic investigation was performed for the $5 d$ impurities in $\mathrm{Fe}, \mathrm{Co}$, and Ni. The preceding paper (part I) (Ref. 7) gives a survey of the current experimental situation. For a quantitative account of the effect detailed electronic structure calculations are necessary. Although no ab initio calculations of the SO-EFG have been reported so far, the potential for such calculations exists: For the theoretical treatment of the SOC in magnetic transition metals several advanced schemes are available, ${ }^{8-10}$ and EFG's in noncubic transition metals can moderately well be reproduced by $a b$ initio calculations. $^{11-13}$

However, the understanding of some elementary relations between the noncubic charge distribution, the SOC, and the band structure will also be necessary for the interpretation of the experimental and theoretical results. These relations are not directly evident from the very generally formulated equations of ab initio calculations. Therefore, we present in this work an approximate but more transparent treatment of the SOC within the tight-binding model. The results are compared with preliminary band structure calculations including SOC. Detailed electronic structure calculations, better representing the impurity systems, are left for future work.

Tight-binding models were already used in the first studies of the SO-EFG to explain the effect: Aiga and Itoh assumed a rigid shift of the partial densities of states by the SOC. ${ }^{14}$ Using this approximation the orbital moment and the SO-EFG can be expressed in terms of the SOC strength, the density of states at the Fermi energy, and the derivative of the density of states at the Fermi energy. This model was extended by Gehring and Williams by the introduction of the crystal potential to treat a possible dependence on the direction of the magnetization. ${ }^{15,16}$ Because of its conceptual simplicity, the rigid shift of the partial densities of states is still used today for qualitative considerations. ${ }^{8,17}$ But this model is in several respects not realistic for transition metals, as discussed in detail in Appendix A.

Demangeat has used the tight-binding approximation in 
combination with realistic band structures to calculate the SO-EFG at impurities in $\mathrm{Fe}$ and Ni. ${ }^{18,19}$ The emphasis of this pioneering work, however, was on the attempt of a quantitative calculation of the SO-EFG and the equations used were not simple enough for qualitative considerations.

The expressions for the noncubic charge distribution that are used in this work are both transparent enough to reveal the essential relationships and realistic enough to treat transition-metal band structures. They are obtained by applying the tight-binding approximation and the perturbation theory to the scattering of the conduction electrons by the $\mathrm{SOC}$ at an impurity. The derivation of these model expressions is discussed in detail in Sec. II.

The final equations are, however, not as simple as those for the rigid shift of the partial densities of states. A detailed discussion is necessary to relate the properties of the spinorbit effects to the features of the band structure. The orbital moment and the noncubic charge distribution are discussed in Secs. III and IV, respectively. The discussion of the orbital moment as an effect of first order in the SOC is included there because it makes the more involved discussion of the noncubic charge distribution, a second-order effect, more transparent.

The conclusions in Secs. III and IV are not restricted to a particular system. In Sec. V a special class of systems, the $5 d$ impurities in $\mathrm{Fe}$, are investigated. This is mainly motivated by the fact that at present most of the available experimental data are for these systems. In addition, their band structure shows some peculiar features that deserve a separate discussion.

\section{TIGHT-BINDING ANALYSIS}

The band structure of the system without SOC is assumed to be already known. To investigate the consequences of the SOC the following three approximations are introduced.

The first approximation is the tight-binding model: The conduction electron states $\psi_{i}$ are described as linear combinations of the atomiclike orbitals $\phi_{j \vec{R}}$ located at the lattice sites $\vec{R}$ :

$$
\psi_{i}=\frac{1}{\sqrt{N}} \sum_{j, \vec{R}} a_{i j \vec{R}} \phi_{j \vec{R}} .
$$

The index $j$ runs over the orbitals at each lattice site and is confined to the ten $d$ orbitals of the outermost $d$ shell. In a homogeneous system the coefficients $a_{i j \vec{R}}$ depend on $\vec{R}$ only in the form of the phase factor $\exp (i \vec{k} \cdot \vec{R})$. In the vicinity of an impurity the amplitude may also vary. In any case, we will not make explicit use of the $a_{i j \vec{R}}$. We will use instead the local density of states $\rho_{m n}(e)$ at the impurity site $\vec{R}=0$ :

$$
\rho_{m n}(e)=\sum_{i}\left\langle\phi_{m 0} \mid \psi_{i}\right\rangle\left\langle\psi_{i} \mid \phi_{n 0}\right\rangle \delta\left(e-e_{i}\right) .
$$

The expectation value of an operator $O$ at the impurity site is given by the following trace:

$$
\langle O\rangle=\int^{e_{F}} \operatorname{Tr}[O \rho(e)] d e,
$$

where $e_{F}$ is the Fermi energy.

The second approximation is to neglect the SOC in the host. This seems to be primarily justified only for heavy impurities, where the SOC is an order of magnitude larger than in the $\mathrm{Fe}$ or $\mathrm{Ni}$ host. However, most of the available SO-EFG data are for the $5 d$ impurities. Furthermore, it is shown in Appendix B that similar equations are obtained for the pure host. Therefore, most of the conclusions are expected to apply even if the SOC's of host and impurity are of comparable magnitude.

The SOC represents thus an additional potential $\Delta V^{(S O)}$ at the impurity site. This is, however, a well-known scattering problem. An elegant solution is provided by the Green's function formalism ${ }^{20,21}$ :

$$
\begin{gathered}
G(e)=\mathcal{P}\left[\int \frac{\rho\left(e^{\prime}\right)}{\left(e-e^{\prime}\right)} d e^{\prime}\right]-i \pi \rho(e), \\
\rho(e)=-(1 / \pi) \operatorname{Im}[G(e)], \\
G(e)=G^{(0)}(e)+G^{(0)}(e) \Delta V^{(S O)} G(e) .
\end{gathered}
$$

$G(e)$ is the Green's function, and $\mathcal{P}$ is the principal part of the integral. $G(e)$ and $\rho(e)$ are closely related and can easily be converted into each other via Eqs. (4) and (5). Equation (6) is the Green's function formulation of the scattering problem: The Green's function $G(e)$ of the system with $\Delta V^{(S O)}$ is expressed in terms of $\Delta V^{(S O)}$ and the Green's function $G^{(0)}(e)$ of the system without $\Delta V^{(S O)}$.

Equations (4)-(6) are in principle operator equations. Because of the tight-binding approximation and the localization of $\Delta V^{(S O)}$, however, $\rho(e), G(e), G^{(0)}(e), \Delta V^{(S O)}$, and $O$ are $10 \times 10$ matrices acting on the ten $d$ orbitals at the impurity site. Thus Eq. (6) is a set of linear equations, which has to be solved for each energy $e$.

The third approximation is to treat $\Delta V^{(S O)}$ in perturbation theory. Equation (6) is still not transparent since the unknown quantity $G(e)$ enters both sides of the equation. This can be removed by expanding the Green's function in powers of $\Delta V^{(S O)}$ :

$$
\begin{aligned}
G= & G^{(0)}+G^{(0)} \Delta V^{(S O)} G^{(0)} \\
& +G^{(0)} \Delta V^{(S O)} G^{(0)} \Delta V^{(S O)} G^{(0)}+\cdots .
\end{aligned}
$$

This should be a reasonable approximation as long as the SOC is considerably smaller than the bandwidth.

The next step is to find explicit expressions for $\rho^{(0)}$, $\Delta V^{(S O)}$, and $\hat{O}$ to substitute into Eqs. (3), (5), and (7). We introduce first the following notation to distinguish between the two reference frames that enter our problem: $x, y$, and $z$ denote the principal axes of the cubic lattice and $x^{\prime}, y^{\prime}$, and $z^{\prime}$ denote an axes system where the $z^{\prime}$ direction is parallel to the magnetization.

The ten $d$ orbitals are represented by a basis set where the spin is parallel (spin up, $\uparrow$ ) or antiparallel (spin down, $\downarrow$ ) to the magnetization and the angular-dependent part behaves like $x y, y z, z x, x^{2}-y^{2}$, or $3 z^{2}-r^{2}{ }^{22}$ Here $x y, y z$, and $z x$ are the $t_{2 g}$ orbitals (other notation: $\epsilon, \Gamma_{5}, \Gamma_{25}^{\prime}$ ), and $x^{2}$ $-y^{2}$ and $3 z^{2}-r^{2}$ the $e_{g}$ orbitals (other notation: $\gamma, \Gamma_{3}$, 
$\left.\Gamma_{12}\right)$. The former are preferentially oriented along the [110] directions, the latter along the [100] directions. $\rho^{(0)}(e)$ is diagonal in this representation with only four different matrix elements $\rho_{e_{g}}^{\uparrow}(e), \rho_{t_{2 g}}^{\uparrow}(e), \rho_{e_{g}}^{\downarrow}(e)$, and $\rho_{t_{2 g}}^{\downarrow}(e)$. The radial part of the orbitals is assumed to be independent of the energy of the electron and the type of $d$ orbital. It enters only in the form of the SOC strength $\xi$ and the average $\left\langle 1 / r^{3}\right\rangle$.

$\Delta V^{(S O)}$ is given by $\vec{\xi} \vec{s} \cdot \vec{l}$ acting on the $d$ orbitals at the impurity site. The matrix elements of $\Delta V^{(S O)}$ in the used representation are given, for example, in Refs. 18 and 23.

The expectation values that are investigated in this work are $\left\langle l_{z^{\prime}}\right\rangle$ as a measure for the orbital moment and $\left\langle l_{z^{\prime}}^{2}-l(l\right.$ $+1) / 3\rangle$ as a measure for the deviation of the charge distribution from cubic symmetry. For $d$ electrons the latter quantity is connected to the $z^{\prime} z^{\prime}$ component of the EFG by ${ }^{7}$

$$
V_{z^{\prime} z^{\prime}}=(2 / 7) e\left\langle 1 / r^{3}\right\rangle\left\langle l_{z^{\prime}}^{2}-\frac{l(l+1)}{3}\right\rangle .
$$

We need to consider only the $z^{\prime}$ components, since in practice only these are accessible to the experiment.

If we now combine Eqs. (3), (5), and (7), we obtain in lowest nonvanishing order [first-order perturbation theory for $\left\langle l_{z^{\prime}}\right\rangle$ and second order for $\left.\left\langle l_{z^{\prime}}^{2}-l(l+1) / 3\right\rangle\right]$ :

$$
\begin{aligned}
\left\langle l_{z^{\prime}}\right\rangle=-\frac{1}{\pi} \operatorname{Im} \int & { }^{e_{F}} \operatorname{Tr}\left[\left(l_{z^{\prime}}\right) G^{(0)}(e)(\xi \vec{s} \cdot \vec{l}) G^{(0)}(e)\right] d e, \\
\left\langle l_{z^{\prime}}^{2}-\frac{l(l+1)}{3}\right\rangle= & -\frac{1}{\pi} \operatorname{Im} \int{ }^{e_{F}} \operatorname{Tr}\left[\left(l_{z^{\prime}}^{2}-\frac{l(l+1)}{3}\right) G^{(0)}(e)\right. \\
& \left.\times(\overrightarrow{\xi s} \cdot \vec{l}) G^{(0)}(e)(\xi \vec{s} \cdot \vec{l}) G^{(0)}(e)\right] d e .(10)
\end{aligned}
$$

If the matrix multiplications in Eq. (9) are carried out, the following expression for the orbital moment is obtained:

$$
\begin{gathered}
\left\langle l_{z^{\prime}}\right\rangle=\xi \int^{e_{F}} \sum_{s t} \sum_{i j} b^{s t} c_{i j} \Omega_{i j}^{s t}(e) d e, \\
\Omega_{i j}^{s t}=-(1 / \pi) \operatorname{Im}\left[G_{i}^{s}(e) G_{j}^{t}(e)\right] .
\end{gathered}
$$

The $G_{i}^{s}$ s are the matrix elements of $G^{(0)}(e)$. The superscripts $s$ and $t$ denote $\uparrow$ or $\downarrow$ spin, the subscripts $i$ and $j, e_{g}$ or $t_{2 g}$ orbitals. st is summed over $\uparrow \uparrow$ and $\downarrow \downarrow$ and the $b^{s t}$,s are

$$
b^{\uparrow \uparrow}=1, \quad b^{\downarrow \downarrow}=-1 .
$$

$i j$ is summed over $e_{g} t_{2 g}$ and $t_{2 g} t_{2 g}$ and the $c_{i j}$ 's are

$$
c_{e t}=4, \quad c_{t t}=1,
$$

where $e_{g}$ and $t_{2 g}$ is abbreviated by $e$ and $t$.

We obtain from Eq. (10) for the noncubic charge distribution

$$
\left\langle l_{z^{\prime}}^{2}-\frac{l(l+1)}{3}\right\rangle=\xi^{2} \int^{e_{F}} \sum_{s t u} \sum_{i j k} b^{s t u} c_{i j k} \Omega_{i j k}^{s t u}(e) d e,
$$

$$
\Omega_{i j k}^{s t u}=-(1 / \pi) \operatorname{Im}\left[G_{i}^{s}(e) G_{j}^{t}(e) G_{k}^{u}(e)\right] .
$$

stu is summed over $\uparrow \uparrow \uparrow, \uparrow \downarrow \uparrow, \downarrow \uparrow \downarrow$, and $\downarrow \downarrow \downarrow$. The respective $b^{s t u}$ 's are

$$
\begin{aligned}
& b^{\uparrow \uparrow \uparrow}=1, \quad b^{\uparrow \downarrow \uparrow}=-1, \\
& b^{\downarrow \uparrow \downarrow}=-1, \quad b^{\downarrow \downarrow \downarrow}=1 .
\end{aligned}
$$

$i j k$ is summed over $e_{g} t_{2 g} e_{g}, e_{g} t_{2 g} t_{2 g}, t_{2 g} e_{g} t_{2 g}$, and $t_{2 g} t_{2 g} t_{2 g}$. The $c_{i j k}$ 's depend on the direction of the magnetization:

$$
\begin{gathered}
c_{e t e}=2-2 F(\vec{\alpha}), \quad c_{e t t}=2 F(\vec{\alpha}), \\
c_{t e t}=2-F(\vec{\alpha}), \quad c_{t t t}=-\frac{1}{2}+F(\vec{\alpha}), \\
F(\vec{\alpha})=3\left(\alpha_{x}^{2} \alpha_{y}^{2}+\alpha_{y}^{2} \alpha_{z}^{2}+\alpha_{z}^{2} \alpha_{x}^{2}\right) .
\end{gathered}
$$

$\vec{\alpha}$ is the unit vector parallel to the direction of the magnetization.

Each Green's function $G_{i}^{s}(e)$ is, as defined by the right side of Eq. (4), simply a linear combination of the respective partial density of states $\rho_{i}^{s}\left(e^{\prime}\right)$ at different energies $e^{\prime}$. Thus, the sets of equations (11)-(14) and (15)-(19) express the orbital moment and the noncubic charge distribution directly in terms of $\xi$ and the four partial densities of states $\rho_{e_{g}}^{\uparrow}(e)$, $\rho_{t_{2 g}}^{\uparrow}(e), \rho_{e_{g}}^{\downarrow}(e)$, and $\rho_{t_{2 g}}^{\downarrow}(e)$.

The equations are used in the following sections in different ways: (i) The basic relations between orbital moment, noncubic charge distribution, and band structure are derived from the structure of the equations. This structure mainly lies within the $b$ and $c$ coefficients and the definition of the $\Omega$ 's. Therefore, it is not immediately obvious from the Eq. (11) or (15). But it will become clear from the discussion of these quantities in Secs. III and IV. (ii) The equations are evaluated for simple model densities of states to study directly the influence of particular features of the density of states. (iii) Applied to the $e_{g}$ and $t_{2 g}$ densities of states of a particular system the equations yield approximate results for the orbital moment and the noncubic charge distribution. The main trends should be reproduced in this way although no complete agreement with more precise calculations can be expected.

The advantage of the proposed tight-binding analysis with respect to other perturbation treatments of the SOC is that the band structure enters the equations in the form of four partial densities of states, a comparatively transparent form. This is a feature of the impurity problem, because the scattering by the localized SOC is wave vector independent and allows us to combine all states with the same energy to the local density of states. On the contrary, the band structure enters most other perturbation treatments in the form of a set of eigenstates and eigenenergies for each wave vector. It is impossible to obtain a qualitative understanding of the band structure in this detailed form. The equations for the impurity problem are thus easier to understand than the equations for the pure metal.

Equation (6) was already used in Ref. 15 to investigate the orbital moment and the noncubic charge distribution. The decisive improvement with respect to this work is that we 
transform the implicit equation for $G(e)$ into the explicit equation (7) by using perturbation theory. The explicit form decisively facilitates the interpretation of the structure of the equations. Explicit equations were also derived in Ref. 15, but under the assumption of a Lorentzian shape of the densities of states. This assumption is far less realistic than the perturbation theory.

Apart from the approximations that are used in the derivation of our model we want to mention also some more fundamental limitations: (i) We neglect in this work the $p$ electrons. However, it is well known that they can make an essential contribution to the EFG even if the electronic structure is dominated by $d$ electrons, because the radial part of $p$ and $d$ orbitals is very different. ${ }^{11}$ Whether there is a sizable $p$ contribution to the SO-EFG will depend on the number of $p$ and $d$ electrons and on the respective radial matrix elements $\xi$ and $\left\langle 1 / r^{3}\right\rangle$ in the particular case. (ii) It is now commonly assumed that, according to Hund's second rule, the orbital moment is enhanced by intraatomic correlations by up to a factor of 2. In recent $a b$ initio calculations these correlations are taken into account by an additional "orbital polarization" (OP) term. ${ }^{24,25}$ This "OP mechanism" is not taken into account in our work. Its importance for $4 d$ and $5 d$ elements and for the noncubic charge distribution remains to be investigated.

\section{ORBITAL MOMENT}

\section{A. Competition between band structure and SOC}

The quenching of the orbital moment in transition metals is the result of the competition between the mixing of the states by the SOC and the splitting of the states by the band structure: ${ }^{26}$ The conduction electron states are split into a set of bands. The energy separation is of the order of the bandwidth $W$. The expectation value of the orbital moment would vanish for all eigenstates in the absence of the SOC. The SOC tends to mix the states of each spin direction into eigenstates of $l_{z^{\prime}}$, but the mixing is hindered by the splitting of the states.

The structure of the quantity $\Omega_{i j}^{s t}(e)$, the basic element on the right side of Eq. (11), provides a more formal description of this quenching mechamism. $\Omega_{i j}^{s t}(e)$ is the density of orbital moment per unit energy $d\left\langle l_{z^{\prime}}\right\rangle / d e$ at the energy $e$ due to the mixing of ${ }_{i}^{s}$ and ${ }_{j}^{t}$ states by the SOC. For simplicity, we consider first only $\uparrow$ states and neglect the difference between $e_{g}$ and $t_{2 g}$ states. That means $\rho_{e_{g}}=\rho_{t_{2 g}}=(1 / 5) \rho_{t}$, where $\rho_{t}$ is the total density of $d$ states. Using Eqs. (4), (11), and (12) we can express the density of orbital moment as a function of the density of states:

$$
\left(d\left\langle l_{z^{\prime}}\right\rangle / d e\right)^{\uparrow \uparrow}(e)=(2 / 5) \xi \rho_{t}^{\uparrow}(e) \mathcal{P} \int \frac{\rho_{t}^{\uparrow}\left(e^{\prime}\right)}{\left(e-e^{\prime}\right)} d e^{\prime} .
$$

The structure of this expression reflects the quenching of the orbital moment by the energy distribution of the states: The induced moment is proportional to $\rho(e) \rho\left(e^{\prime}\right)$, the product of the number of the involved states at $e$ and $e^{\prime}$. The various matrix elements are absorbed in the prefactor $2 / 5$. The mixing of the states is suppressed by a factor $\xi /\left(e-e^{\prime}\right)$. There-
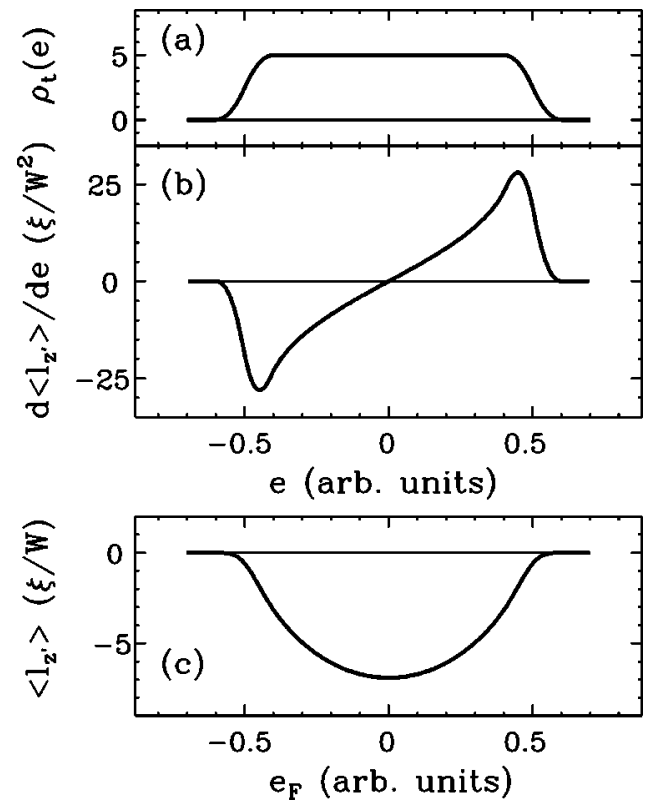

FIG. 1. Orbital moment for a smooth $\uparrow$ band. (a) Density of states. (b) Density of orbital moments. (c) Orbital moment as a function of the Fermi energy. $W$ is the bandwidth.

fore, the orbital moment is of the order $\xi / W$. The sign of the admixtures is determined by the factor $1 /\left(e-e^{\prime}\right)$. This factor leads to the mixing of wave function components with orbital moment parallel to the spin from the energetically lower state to the higher state. In return, components with orbital moment antiparallel to the spin are mixed from the higher state to the lower state. In this way an orbital moment antiparallel to the spin (the energetically favored orientation) is induced in the lower half of the band and an orbital moment parallel to the spin in the upper half of the band. The resulting distribution of the orbital moment in the $\uparrow$ band is shown for an idealized, almost rectangular density of states in Fig. $1(\mathrm{~b})$.

The orbital moment of the system is obtained by the integration of $d\left\langle l_{z^{\prime}}\right\rangle / d e$ up to the Fermi energy. The dependence of the orbital moment on the band filling is shown in Fig. 1(c): Since the states with antiparallel orientation of the orbital moment are filled up first, the orbital moment is always antiparallel to the spin and the maximum moment is found for a half filled band.

The formal expression for the orbital moment as a function of the Fermi energy is obtained by integration of Eq. (20):

$$
\left\langle l_{z^{\prime}}\right\rangle^{\uparrow \uparrow}=(2 / 5) \xi \int^{e_{F}} d e \int_{e_{F}} d e^{\prime} \frac{\rho_{t}^{\uparrow}(e) \rho_{t}^{\uparrow}\left(e^{\prime}\right)}{\left(e-e^{\prime}\right)} .
$$

This particular form is obtained by a rearrangement of the integration limits: Only unoccupied states need to be considered for $e^{\prime}$, since admixtures between occupied states do not change the total orbital moment.

We will extend in the following the discussion from a band with uniform spin direction and almost rectangular density of states to a realistic ferromagnetic band structure by the following steps: (i) Both spin directions are taken into 


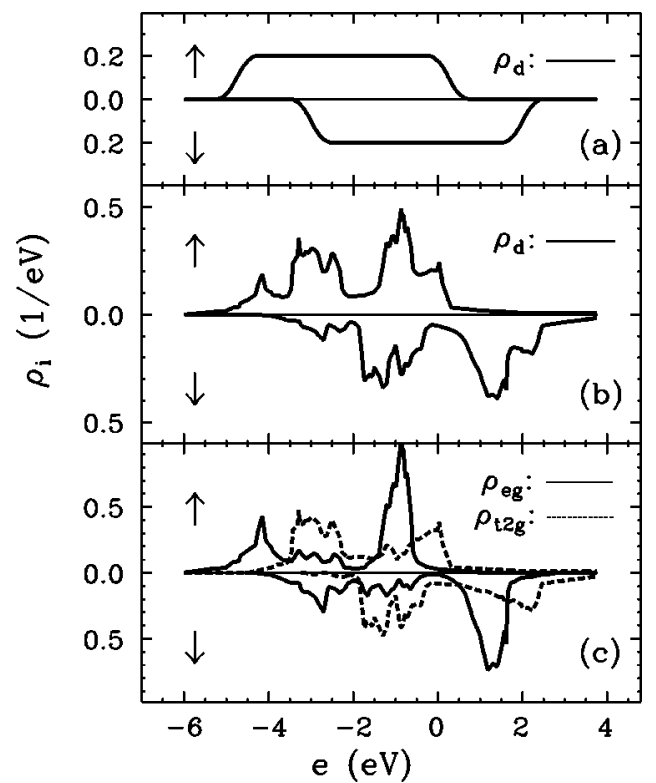

FIG. 2. Model densities of states for Fe. (a) Smooth density of states $\left(\rho_{d}=1 / 5 \rho_{t}\right)$. (b) Realistic density of states; $e_{g}$ and $t_{2 g}$ states are not distinguished. (c) Realistic density of states; $e_{g}$ and $t_{2 g}$ states are distinguished. The densities in (b) and (c) were taken from Ref. 8.

account. (ii) The rectangular band is replaced by a realistic density of states. (iii) $e_{g}$ and $t_{2 g}$ states are distinguished. For each of these steps the density of states and the dependence of $\left\langle l_{z^{\prime}}\right\rangle$ on the band filling are shown in Figs. 2 and 3, respectively. The partial densities of states for Fe from Ref. 8 were chosen as the example for a realistic ferromagnetic band structure.

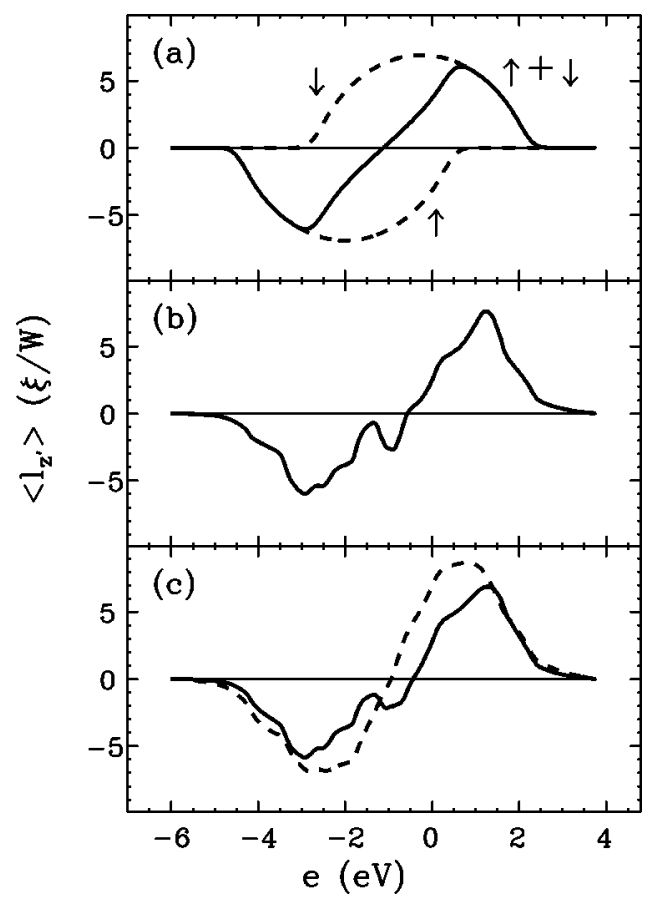

FIG. 3. Orbital moment as a function of the Fermi energy for the densities of states from Fig. 2. Dashed lines in (a): contributions from the $\uparrow$ and the $\downarrow$ band. Dashed line in (c): orbital moment according to Eq. (26).

\section{B. Competition between $\uparrow$ and $\downarrow$ band}

We have just discussed the orbital moment of the $\uparrow$ band, which arises from the mixing of the $\uparrow$ states. To this we have to add the orbital moment of the $\downarrow$ band, which arises from the mixing of the $\downarrow$ states. It essentially shows the same behavior. But the relevant densities are those of the $\downarrow$ band and it has the opposite sign since it is also antiparallel to the spin. The competition between the contributions from the $\uparrow$ and $\downarrow$ bands is formally described by the $b$ coefficients in Eq. (11): With respect to the spin indices $s, t$ of the $\Omega_{i j}^{s t}$, s we can distinguish a $\uparrow \uparrow$ and a $\downarrow \downarrow$ contribution, which are added in the form $\uparrow \uparrow-\downarrow \downarrow$.

This partial cancellation between the orbital moments of the $\uparrow$ and the $\downarrow$ band is important for the dependence of the total orbital moment on the band filling. In the paramagnetic state, $\uparrow$ and $\downarrow$ bands are identical and the cancellation is exact. In a "simple" ferromagnetic band structure, $\uparrow$ and $\downarrow$ bands are just shifted with respect to each other by the exchange splitting. The $\downarrow \downarrow$ and $\uparrow \uparrow$ terms are, as a function of $e_{F}$, accordingly also just shifted with respect to each other by the exchange splitting. The typical dependence of the orbital moment as the difference of both terms on the band filling is shown in Fig. 3(a). This dependence is in accordance with Hund's third rule: The orbital moment is parallel to the magnetization for a more than half filled band and antiparallel for a less than half filled band.

The absence of $\downarrow \uparrow$ and $\uparrow \downarrow$ terms directly arises from the form of Eq. (9). The matrix elements in the trace are found to have the following form:

$$
\left\langle i\left|l_{z^{\prime}}\right| j\right\rangle\langle j|\vec{\xi} \vec{s}| i\rangle,
$$

where $i$ and $j$ denote the mixed states. Since $l_{z^{\prime}}$ does not change the spin, only the following two combinations are possible with respect to the spins of the involved states:

$$
\left\langle\uparrow\left|l_{z^{\prime}}\right| \uparrow\right\rangle\left\langle\uparrow\left|l_{z^{\prime}}\right| \uparrow\right\rangle
$$

and

$$
-\left\langle\downarrow\left|l_{z^{\prime}}\right| \downarrow\right\rangle\left\langle\downarrow\left|l_{z^{\prime}}\right| \downarrow\right\rangle,
$$

which both arise from the $s_{z^{\prime}} l_{z^{\prime}}$ component of the SOC. The $\left(s_{+}, l_{-},+s_{-}, l_{+}\right)$component of the SOC also admixes $\uparrow$ and $\downarrow$ states. But this does not contribute to $\left\langle l_{z^{\prime}}\right\rangle$ in firstorder perturbation theory.

\section{Sensitivity to band structure details}

Figure 3(b) shows the orbital moment if we replace the rectangular densities of states by realistic ones. The dependence on the band filling is still rather smooth and remarkably similar to the case of the rectangular densities of states. This shows that the orbital moment is rather insensitive to band structure details. It is to a large extent already determined by the relative positions of the $\uparrow$ and $\downarrow$ band, the position of the Fermi energy, and the ratio $\xi / W$.

A further band structure detail is the distinction between the $e_{g}$ and $t_{2 g}$ densities of states. The $c$ coefficients in Eq. (11) describe to which extent mixings between $e_{g}$ states, between $t_{2 g}$ states, and between $e_{g}$ and $t_{2 g}$ states contribute to 
the orbital moment: With respect to the $i j$ indices of the $\Omega_{i j}^{s t}$ 's we find $e_{g} t_{2 g}$ and $t_{2 g} t_{2 g}$ terms, which are added in the form $4 e_{g} t_{2 g}+t_{2 g} t_{2 g}$. This particular set of $c$ coefficients is the result of the matrix elements of the SOC and $l_{z}$, between the various $e_{g}$ and $t_{g}$ orbitals.

Figure 3(c) shows the orbital moment if $e_{g}$ and $t_{2 g}$ states are distinguished. The orbital moment is obviously only slightly affected by this distinction. This is in accordance with the general insensitivity to band structure details.

\section{Expansion in directional cosines}

We have shown above that only the $s_{z^{\prime}} l_{z^{\prime}}$ component of $\xi \vec{s} \cdot \vec{l}$ contributes to the orbital moment. If we express $l_{z^{\prime}}$ as $\sum_{i} \alpha_{i} l_{i}$, where $\vec{\alpha}$ is the unit vector in the direction of the magnetization, Eq. (9) can be written in the form

$$
\left\langle l_{z^{\prime}}\right\rangle=\sum_{i j} d_{i j} \alpha_{i} \alpha_{j}
$$

The summation over $i$ and $j$ is over the cubic coordinates $x$, $y$, and $z$. The orbital moment is thus a polynomial of second order in the directional cosines $\alpha_{i}$. All matrix elements and details of the band structure are put into the coefficients $d_{i j}$. The important point is that these obey the same symmetry as the system without SOC. Equation (23) provides, therefore, a particularly transparent formulation of some well-known symmetry properties of the orbital moment.

For example, the orbital moment in a cubic lattice is independent of the direction of the magnetization, because any second-order polynomial of cubic symmetry has the form

$$
d\left(\alpha_{x}^{2}+\alpha_{y}^{2}+\alpha_{z}^{2}\right)=d
$$

The isotropy of the orbital moment can thus be traced back to the combination of the cubic lattice symmetry with the twofold appearance of the orbital moment operator in the first-order expression for $\left\langle l_{z^{\prime}}\right\rangle$. In fact, the orbital moment depends in a noncubic lattice and/or in higher-order perturbation theory in general on the direction of the magnetization. ${ }^{27}$

At this point the question may arise how the orbital moment can become anisotropic in higher order when it is in first order still completely isotropic. The answer is that the induced orbital current is actually in first order not completely isotropic. It is, namely, only the expectation value of the orbital moment, $\left\langle l_{z^{\prime}}\right\rangle$, that is isotropic. But the angular distribution of the orbital current around the nucleus can be shown to depend in general already in first order on the direction of the magnetization.

A further symmetry property is that only odd orders can contribute to the orbital moment in the perturbation expansion. This follows from the inversion symmetry of the unperturbed system which allows nonvanishing coefficients only in front of an even number of $\alpha_{i}$ 's. The next term beyond first order is thus obtained in third order and is proportional to $(\xi / W)^{3} \cdot{ }^{27}$

\section{E. Simplified model}

Every model is a compromise between simplicity and transparency on the one side and realism on the other side.
Therefore, we offer here also a much simpler model version of the analysis presented above.

Motivated by the insensitivity of the orbital moment to band structure details, these details are neglected by replacing the energy splitting $\left(e-e^{\prime}\right)$ in Eq. (21) by an appropriate average $\Delta e$. The right side of Eq. (21) is then the product of the number of all occupied states,

$$
N_{o}=\int^{e_{F}} \rho_{t}\left(e^{\prime}\right) d e^{\prime},
$$

the number of all unoccupied states,

$$
N_{u}=\int_{e_{F}} \rho_{t}\left(e^{\prime}\right) d e^{\prime}=5-N_{o},
$$

the ratio $\xi /(\Delta e)$, and a numerical prefactor. If we approximate $1 / \Delta e$ by

$$
\left(4 / W^{2}\right) \int_{0}^{W / 2} d e \int_{W / 2}^{W} d e^{\prime} \frac{1}{\left(e-e^{\prime}\right)},
$$

we obtain $1 / \Delta e \approx-4 / W$. By applying this procedure also to the orbital moment of the $\downarrow$ band we obtain for the total orbital moment

$$
\left\langle l_{z^{\prime}}\right\rangle \approx(8 / 5)(\xi / W)\left[-N_{o}^{\uparrow}\left(5-N_{o}^{\uparrow}\right)+N_{o}^{\downarrow}\left(5-N_{o}^{\downarrow}\right)\right] .
$$

Oviously, Eq. (26) is much simpler than Eq. (11), but also less realistic and flexible. For example, a separation of the band in two parts, as found for the $5 d$ impurities in $\mathrm{Fe}$, is not provided for by the parameters in Eq. (26). Nevertheless, it will in many cases already describe the major trend of the orbital moment.

The orbital moment according to Eq. (26) is shown as the dashed line in Fig. 3(c). With respect to the full expression (11) [solid line in Fig. 3(c)] the variation with band filling as well as the absolute magnitude of the effect is moderately well reproduced.

Table I compares the prediction of Eq. (26) for the orbital moments of $\mathrm{Fe}, \mathrm{Co}$, and $\mathrm{Ni}$ with the experiment and with the results of fully relativistic $a b$ initio calculations with and without the OP mechanism. We find again that Eq. (26) not only reproduces the order of magnitude, but also the main systematic trend. Of course, there can be no close quantitative agreement since we have used, for example, $\xi$ 's that were calculated for the free atom. Table I also shows that the OP mechanism, which is not taken into account in the present work, can be quite important.

\section{NONCUBIC CHARGE DISTRIBUTION}

In contrast to the orbital moment, the noncubic charge distribution arises only in second-order perturbation theory. Because of the higher order, there is no direct correspondence to the orbital moment and the dependence on the band structure is more complex.

The higher order can be understood in the following way: The eigenstates of the system in the absence of the SOC contain components with positive and negative orbital mo- 
TABLE I. Orbital moments of Fe, Co, and Ni. (mod) refers to Eq. (26), (SO) and (OP) refer to ab initio calculations without and with orbital polarization, and (exp) refers to the experiment. The parameters $W, N_{o}^{\uparrow}$, and $N_{o}^{\downarrow}$ are rough estimates.

\begin{tabular}{lcccccccc}
\hline \hline & $\xi(\mathrm{eV})^{\mathrm{a}}$ & $W(\mathrm{eV})$ & $N_{o}^{\uparrow}$ & $N_{o}^{\downarrow}$ & $l_{z^{\prime}}^{(\bmod )}$ & $l_{z^{\prime}}^{(\text {SO)b }}$ & $l_{z^{\prime}}^{(\text {OP)b }}$ & $l_{z^{\prime}}^{(\text {exp }) \mathrm{b}}$ \\
\hline $\mathrm{Fe}$ & 0.054 & 6.2 & 4.8 & 2.6 & 0.074 & 0.048 & 0.078 & 0.08 \\
$\mathrm{Co}$ & 0.068 & 5.8 & 5.0 & 3.4 & 0.102 & 0.076 & 0.123 & 0.14 \\
$\mathrm{Ni}$ & 0.086 & 5.1 & 5.0 & 4.4 & 0.071 & 0.048 & 0.066 & 0.05 \\
\hline \hline
\end{tabular}

${ }^{\mathrm{a}}$ Reference 28 .

${ }^{\mathrm{b}}$ Reference 25 .

ments to exactly the same extent. The mixing of the states by the SOC leads to a growth of the components of one orbital moment direction. But at the same time the respective components with the opposite orbital moment diminish, in firstorder perturbation theory to exactly the same extent. Since orbitals with opposite orbital moments have the same spatial electron distribution, the charge distribution does not change in first-order perturbation theory.

We neglect in the following magnetostriction as a source of the noncubic charge distribution. This point is discussed in Appendix C.

\section{A. Competition between band structure and SOC}

The quenching of the spin-orbit induced mixing of states by the band structure also dominates the physics of the noncubic charge distribution. The structure of $\Omega_{i j l}^{s t u}(e)$, the basic element on the right side of Eq. (15), provides the formal description of this quenching.

$\Omega_{i j l}^{s t u}(e)$ is the density of noncubic charge distribution per unit energy $d\left\langle l_{z^{\prime}}^{2}-l(l+1) / 3\right\rangle / d e$ at the energy $e$ due to the mixing of ${ }_{i}^{s},{ }_{j}^{t}$, and ${ }_{l}^{u}$ states by the SOC. There are 14 different $\Omega_{i j l}^{s t u}(e)$ 's, according to whether $\uparrow$ or $\downarrow, e_{g}$ or $t_{2 g}$ states are mixed. For simplicity, we again first consider only $\uparrow$ states and assume $\rho_{e_{g}}(e)=\rho_{t_{2 g}}(e)=(1 / 5) \rho_{t}^{\uparrow}(e)$. Using Eqs. (4), (15), and (16), we can then express the noncubic charge distribution in the following way as a function of the density of states:

$$
\begin{gathered}
\left(d\left(l_{z^{\prime}}^{2}-\frac{l(l+1)}{3}\right) / d e\right)^{\uparrow \uparrow \uparrow}(e) \\
=(7 / 250) \xi^{2}\left[3 \rho_{t}^{\uparrow}(e) \mathcal{P} \iint \frac{\rho_{t}^{\uparrow}\left(e^{\prime}\right) \rho_{t}^{\uparrow}\left(e^{\prime \prime}\right)}{\left(e-e^{\prime}\right)\left(e-e^{\prime \prime}\right)} d e^{\prime} d e^{\prime \prime}\right. \\
\left.-\pi^{2} \rho_{t}^{\uparrow}(e) \rho_{t}^{\uparrow}(e) \rho_{t}^{\uparrow}(e)\right] .
\end{gathered}
$$

The structure of Eq. (27) reflects that the noncubic charge distribution arises from twofold admixtures by the SOC: The effect is proportional to the triple product of the involved densities of states $\rho(e) \rho\left(e^{\prime}\right) \rho\left(e^{\prime \prime}\right)$. It is suppressed by a factor $\xi^{2} /\left[\left(e-e^{\prime}\right)\left(e-e^{\prime \prime}\right)\right]$ and scales, therefore, with $(\xi / W)^{2}$. Its distribution over the band is determined by the sign of the factor $1 /\left[\left(e-e^{\prime}\right)\left(e-e^{\prime \prime}\right)\right]$ : For $e$ near the ends of the band this factor is predominantly positive. In the middle of the band positive and negative contributions tend to cancel each other and the last term in Eq. (27), which is always negative, dominates. With respect to the direction of the magnetization there is thus an oblate deformation of the electron distribution at both ends of the band and a prolate deformation in the middle of the band. Figure 4(b) shows the distribution of the noncubic charge distribution over a rectangular band.

Figure 1(b) shows that the largest orbital moment density is in first-order perturbation theory induced at both ends of the band. Therefore, it is perhaps not surprising that the states with large $\left\langle l_{z^{\prime}}^{2}\right\rangle$ concentrate in second-order perturbation theory just at the ends of the band whereas they are missing in the middle of the band.

The noncubic charge distribution of the system is given by the integral over $d\left\langle l_{z^{\prime}}^{2}-l(l+1) / 3\right\rangle / d e$ up to $e_{F}$. The resulting dependence on the band filling is shown in Fig. 4(c): The net deformation of the electron distribution is oblate for a less than half filled band and prolate for a more than half filled band.
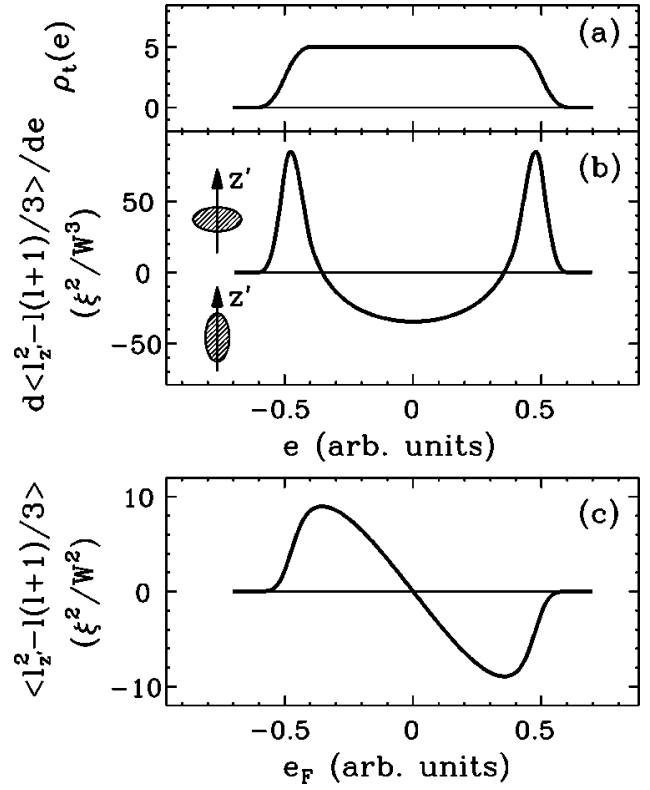

FIG. 4. Noncubic charge distribution for a smooth $\uparrow$ band. (a) Density of states. (b) Density of noncubic charge distribution. (c) Noncubic charge distribution as a function of the Fermi energy. The insets in (b) illustrate that the positive sign corresponds to an oblate deformation of the electron distribution, the negative sign to a prolate deformation. 
For completeness, we give here also the formal expression for the noncubic charge distribution as a function of the density of states:

$$
\begin{aligned}
\left\langle l_{z^{\prime}}^{2}\right. & \left.-\frac{l(l+1)}{3}\right\rangle^{\uparrow \uparrow \uparrow} \\
= & (21 / 250) \xi^{2}\left[\int^{e_{F}} d e \int_{e_{F}} d e^{\prime} \int_{e_{F}} d e^{\prime \prime} \frac{\rho_{t}^{\uparrow}(e) \rho_{t}^{\uparrow}\left(e^{\prime}\right) \rho_{t}^{\uparrow}\left(e^{\prime \prime}\right)}{\left(e-e^{\prime}\right)\left(e-e^{\prime \prime}\right)}\right. \\
& \left.-\int_{e_{F}} d e \int^{e_{F}} d e^{\prime} \int^{e_{F}} d e^{\prime \prime} \frac{\rho_{t}^{\uparrow}(e) \rho_{t}^{\uparrow}\left(e^{\prime}\right) \rho_{t}^{\uparrow}\left(e^{\prime \prime}\right)}{\left(e-e^{\prime}\right)\left(e-e^{\prime \prime}\right)}\right]
\end{aligned}
$$

It is obtained from the integral over Eq. (27) by a rearrangement of the integration limits and by use of the relation

$$
\begin{aligned}
& \mathcal{P}\left(\frac{1}{\left(e-e^{\prime}\right)\left(e-e^{\prime \prime}\right)}+\frac{1}{\left(e^{\prime}-e\right)\left(e^{\prime}-e^{\prime \prime}\right)}+\frac{1}{\left(e^{\prime \prime}-e\right)\left(e^{\prime \prime}-e^{\prime}\right)}\right) \\
& \quad=\pi^{2} \delta\left(e-e^{\prime}\right) \delta\left(e-e^{\prime \prime}\right) .
\end{aligned}
$$

Due to these manipulations, the last term on the right side of Eq. (27) has disappeared and the factor $1 /\left[\left(e-e^{\prime}\right)\left(e-e^{\prime \prime}\right)\right]$ is always positive.

We will now proceed again from a band with uniform spin direction and rectangular density of states to a realistic band structure by the following steps: (i) Both spin directions are taken into account. (ii) A realistic density of states is used. (iii) $e_{g}$ and $t_{2 g}$ states are distinguished. The density of states and the noncubic charge distribution as a function of $e_{F}$ are shown for each of these steps in Figs. 2 and 5, respectively.

\section{B. Competition between $\uparrow$ and $\downarrow$ bands}

The competition between $\uparrow$ and $\downarrow$ bands is also important for the noncubic charge distribution. The $b$ coefficients from Eq. (17) show in which way the $\uparrow$ and the $\downarrow$ states contribute to the effect: With respect to the spin indices stu of the $\Omega_{i j k}^{s t u}$ s we find four types of terms: $\uparrow \uparrow \uparrow, \uparrow \downarrow \uparrow, \downarrow \uparrow \downarrow$, and $\downarrow \downarrow \downarrow$ terms, according to whether three, two, one, or none of the mixed states are $\uparrow$ states. These terms contribute to the total noncubic charge distribution in the form $\uparrow \uparrow \uparrow-\uparrow \downarrow \uparrow-\downarrow \uparrow \downarrow$ $+\downarrow \downarrow \downarrow$. The $\uparrow \uparrow \uparrow$ and $\uparrow \downarrow \uparrow$ terms form the noncubic charge distribution of the $\uparrow$ band, the $\downarrow \downarrow \downarrow$ and $\downarrow \uparrow \downarrow$ terms the noncubic charge distribution of the $\downarrow$ band. We note that via the mixed terms even a full $\uparrow$ band contributes to the effect.

The presence of four terms follows directly from the form of Eq. (10). The matrix elements in the trace are all of the form

$$
\left\langle i\left|l_{z^{\prime}}^{2}-\frac{l(l+1)}{3}\right| j\right\rangle\langle j|\overrightarrow{\xi s} \cdot \vec{l}| k\rangle\langle k|\xi \vec{s} \cdot \vec{l}| i\rangle .
$$

With respect to the spin of the involved states the $s_{z^{\prime}} l_{z^{\prime}}$ component of the SOC gives rise to contributions of the form

$$
\left\langle\uparrow\left|l_{z^{\prime}}^{2}-\frac{l(l+1)}{3}\right| \uparrow\right\rangle\left\langle\uparrow\left|l_{z^{\prime}}\right| \uparrow\right\rangle\left\langle\uparrow\left|l_{z^{\prime}}\right| \uparrow\right\rangle
$$

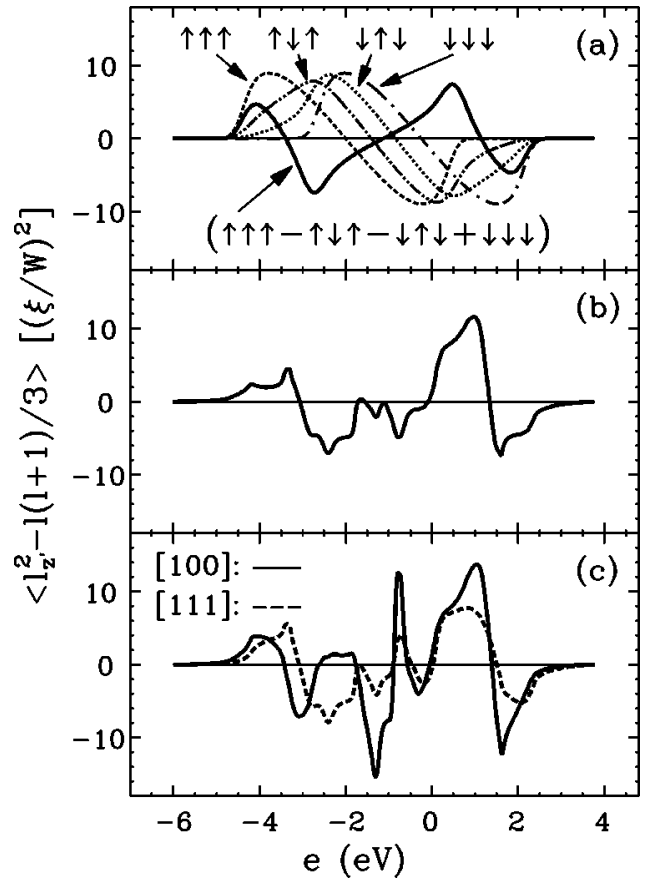

FIG. 5. Noncubic charge distribution as a function of the Fermi energy for the densities of states from Fig. 2. Dashed and dashdotted lines in (a): $\uparrow \uparrow \uparrow, ~ \uparrow \downarrow \uparrow, ~ \downarrow \uparrow \downarrow$, and $\downarrow \downarrow \downarrow$ terms, which are added to the total noncubic charge distribution in the form $\uparrow \uparrow \uparrow$ $-\uparrow \downarrow \uparrow-\downarrow \uparrow \downarrow+\downarrow \downarrow \downarrow$.

or

$$
\left\langle\downarrow\left|l_{z^{\prime}}^{2}-\frac{l(l+1)}{3}\right| \downarrow\right\rangle\left\langle\downarrow\left|l_{z^{\prime}}\right| \downarrow\right\rangle\left\langle\downarrow\left|l_{z^{\prime}}\right| \downarrow\right\rangle,
$$

whereas the $\left(s_{+}, l_{-}, s_{-}, l_{+}\right)$component of the SOC gives rise to contributions of the form

$$
\left\langle\uparrow\left|l_{z^{\prime}}^{2}-\frac{l(l+1)}{3}\right| \uparrow\right\rangle\left\langle\uparrow\left|l_{-^{\prime}}\right| \downarrow\right\rangle\left\langle\downarrow\left|l_{+}\right| \uparrow\right\rangle
$$

or

$$
\left\langle\downarrow\left|l_{z^{\prime}}^{2}-\frac{l(l+1)}{3}\right| \downarrow\right\rangle\left\langle\downarrow\left|l_{+}\right| \uparrow\right\rangle\left\langle\uparrow\left|l_{-,}\right| \downarrow\right\rangle .
$$

The dependence of the total noncubic charge distribution on the band filling can be understood in the following way: The $\uparrow \uparrow \uparrow$ contribution was already discussed above. The other contributions show essentially the same behavior apart from that one, two, or three of the $\uparrow$ densities of states are replaced by $\downarrow$ densities of states. Therefore, there is a relatively smooth transition from $\uparrow \uparrow \uparrow$ to $\uparrow \downarrow \uparrow$ to $\downarrow \uparrow \downarrow$ to $\downarrow \downarrow \downarrow$.

In the paramagnetic state, the $\uparrow$ and $\downarrow$ bands are identical and the various contributions cancel each other exactly. In a "simple" ferromagnet, $\uparrow$ and $\downarrow$ bands are shifted with respect to each other by the exchange splitting. The $\uparrow \uparrow \uparrow, \uparrow \downarrow \uparrow$, $\downarrow \uparrow \downarrow$, and $\downarrow \downarrow \downarrow$ terms are in this case rather similar apart from a gradual shift within this series from the $\uparrow$ to the $\downarrow$ band. The summation of the terms in the form $\uparrow \uparrow \uparrow-\uparrow \downarrow \uparrow-\downarrow \uparrow \downarrow$ $+\downarrow \downarrow \downarrow$ leads to a dependence on $e_{F}$ that resembles the sec- 
ond derivative of one of the terms. The result is that the deformation of the electron distribution changes from oblate to prolate to oblate to prolate as the band is successively filled. The individual contributions and the total noncubic charge distribution are shown in Fig. 5(a) as a function of the band filling.

We can predict from Fig. 5(a) already the sign of the SO-EFG in pure Fe. It should be positive since the $d$ band is somewhat more than half filled. For Ni, where the $d$ band is almost completely filled, we expect a negative SO-EFG.

\section{Sensitivity to band structure details}

Figure 5(b) shows the noncubic charge distribution for a realistic density of states. The comparison with Fig. 5(a) shows the extent of the sensitivity to band structure details: The basic pattern in the dependence on the band filling with the three sign changes remains essentially preserved. But some additional structure is also introduced. The amount of this fine structure corresponds roughly to the amount of fine structure in the density of states.

An essential question for the comparison between band structure calculations and experiment is how accurate the description of the band structure must be. Our results suggest that the reproduction of the main features in the density of states allows already a moderately precise prediction of the noncubic charge distribution. This should be well within the scope of modern $a b$ initio calculations.

Compared to the orbital moment, the sensitivity to band structure details is much larger. This is due to the second order of the effect: The admixtures are weighted by $1 /[(e$ $\left.\left.-e^{\prime}\right)\left(e-e^{\prime \prime}\right)\right]$ instead of $1 /\left(e-e^{\prime}\right)$ and the cancellation between the $\uparrow \uparrow \uparrow, \uparrow \downarrow \uparrow, \downarrow \uparrow \downarrow$, and $\downarrow \downarrow \downarrow$ terms is more complex than between the $\uparrow \uparrow$ and $\downarrow \downarrow$ terms in the case of the orbital moment. Both features increase the importance of the band in the immediate vicinity of the Fermi energy and increase thus the sensitivity to details in this region.

\section{Anisotropy}

The anisotropy of spin-orbit effects in transition metal ferromagnets is due to the following mechanism ${ }^{26,27}$ : The partial densities of states depend on the orientation of the orbitals relative to the lattice. For example, in the case of cubic symmetry, the $e_{g}$ and $t_{2 g}$ orbitals of the $d$ band have different densities of states. The matrix elements of the $s_{z^{\prime}} l_{z^{\prime}}$ and $\left(s_{+}, l_{-}, s_{-}, l_{+},\right)$components of the SOC depend, on the other hand, on the orientation of the orbitals relative to the direction of the magnetization. Therefore, a rotation of the magnetization in the laboratory frame changes the densities of states in the reference frame of the magnetization, whereas the matrix elements of the SOC remain per definition unchanged. This will in general change the magnitude of the spin-orbit effect.

Some conclusions can be drawn at this point: (i) The mechanism is quite general. The anisotropy of spin-orbit effects is accordingly rather the rule and the isotropy of $\left\langle l_{z^{\prime}}\right\rangle$ in cubic symmetry is the exception, as follows also from the discussion in Sec. III D. (ii) The nonspherical symmetry of the band structure that causes the anisotropy comes from the
TABLE II. The $c_{i j k}$ coefficients from Eq. (15) for $M \|[100]$ and $M \|[111] . e_{g}$ is abbreviated by $e$ and $t_{2 g}$ by $t$.

\begin{tabular}{ccccc}
\hline \hline & $c_{\text {ete }}$ & $c_{\text {ett }}$ & $c_{\text {tet }}$ & $c_{t t t}$ \\
\hline$M \|[100]$ & 2 & 0 & 2 & $-1 / 2$ \\
$M \|[111]$ & 0 & 2 & 1 & $1 / 2$ \\
\hline
\end{tabular}

lattice symmetry and not from the SOC. The anisotropy accordingly depends in lowest order only on the band structure and not on the SOC strength. (iii) In view of the distinct differences in the partial densities of states in realistic band structures, large anisotropies can in principle be expected. However, the sensitivity of the particular spin-orbit effect on band structure details plays also a role.

For a more detailed account of the anisotropy we need to know which orbitals contribute to which extent for which direction of the magnetization. The $c$ coefficients in Eq. (15) provide this information: With respect to the $i j k$ indices of the $\Omega_{i j k}^{s t u}$ 's we find $e_{g} t_{2 g} e_{g}, e_{g} t_{2 g} t_{2 g}, t_{2 g} e_{g} t_{2 g}$, and $t_{2 g} t_{2 g} t_{2 g}$ terms, which are weighted by the $c$ coefficients from Eq. (18). The order of $e_{g}$ and $t_{2 g}$ in $i j k$ plays a role when combined with the order of $\uparrow$ and $\downarrow$ in the spin indices stu.

Equation (18) shows that all $c$ coefficients are the sum of an isotropic term and a term proportional to $F(\vec{\alpha})$. The form of the anisotropy is thus given by $F(\vec{\alpha})$, independent of the band structure. $F(\vec{\alpha})$ equals $0,3 / 4$, and 1 for $M \|[100]$, $M \|[110]$, and $M \|[111]$, respectively, and varies rather smoothly between these major directions. The complete dependence on the direction of the magnetization can thus be described by two parameters. This suggest an alternative presentation of the $c$ coefficients: Instead of specifying an isotropic and anisotropic part we can also specify the coefficients for the two extreme cases $M \|[100]$ and $M \|[111]$. The $c$ coefficients are given in this form in Table II.

The $c$ coefficients from Eq. (18) or Table II are the exact answer to the question to which extent the $e_{g}$ and $t_{2 g}$ states contribute for different directions of the magnetization. A less precise but much simpler answer can be deduced from Table II: If one goes from $M \|[100]$ to $M \|[111], 2\left(e_{g} t_{2 g} e_{g}\right)$ is replaced by $2\left(e_{g} t_{2 g} t_{2 g}\right), 2\left(t_{2 g} e_{g} t_{2 g}\right)$ by $\left(t_{2 g} e_{g} t_{2 g}\right)$ $+\left(t_{2 g} t_{2 g} t_{2 g}\right)$, and $-1 / 2\left(t_{2 g} t_{2 g} t_{2 g}\right)$ is left unchanged. To a large part this is the replacement of one $e_{g}$ density of states out of a triple product of densities by a $t_{2 g}$ density of states. Based on this observation, we propose the following rule: Both $e_{g}$ and $t_{2 g}$ orbitals are important for all directions of the magnetization, but the $e_{g}$ orbitals are somewhat more important for $M \|[100]$ and the $t_{2 g}$ orbitals somewhat more for $M \|[111]$.

This interpretation of the $c$ coefficients allows us now to investigate the relation between anisotropy and band structure in more detail.

First, we discuss some limiting cases, which were discussed already in Ref. 15. These are rather unrealistic, but we want to show that the $c$ coefficients from Table II give the same results as the previous work: (i) For $\rho_{e_{g}}=\rho_{t_{2 g}}$ all anisotropic terms vanish and the noncubic charge distribution is isotropic. (ii) If only $e_{g}$ states are important, there is no non- 
cubic charge distribution at all, since $e_{g}$ orbitals alone are not mixed by the SOC. (iii) If only $t_{2 g}$ states are important, only the $t_{2 g} t_{2 g} t_{2 g}$ term does not vanish. The noncubic charge distribution is in this case for $M \|[100]$ and $M \|[111]$ only $-1 / 7$ and $1 / 7$ as large as in the case $\rho_{e_{g}}=\rho_{t_{2 g}}$.

Figure 5(c) shows the [100] and [111] noncubic charge distributions for realistic partial densities of states-namely, those of Fig. 2(c). The variation of the anisotropy with the band filling illustrates the range of anisotropies that can be expected for realistic band structures: Any anisotropy is in principle possible, from no anisotropy to opposite signs of the noncubic charge distribution for $M \|[100]$ and $M \|[111]$. However, in the majority of cases the anisotropy will be larger than $10 \%$, but will not lead to a sign change.

The main trends in the anisotropy will in many cases be explained by the simple rule that the $e_{g}$ states are more important for $M \|[100]$ and the $t_{2 g}$ states for $M \|[111]$. The Fe band structure [Figs. 2(c) and 5(c)] offers two examples: (i) At the bottom of the band $(e=-5 \cdots-3 \mathrm{eV})$, the $t_{2 g}$ band is, with respect to the $e_{g}$ band, shifted to higher energies. Accordingly, in this energy region the dependence of the [111] noncubic charge distribution on $e_{F}$ differs from the respective dependence of the [100] noncubic charge distribution mainly by a shift to higher energies. (ii) In the region $e=-3 \cdots+2 \mathrm{eV}$ the $e_{g}$ band is concentrated in one prominent density of states peak. This leads locally to a small effective $e_{g}$ bandwidth and thus to a large contribution from the $e_{g}$ band. Accordingly, the [100] noncubic charge distribution in this energy region is in most cases considerably larger than the [111] noncubic charge distribution, although the form of the dependence on $e_{F}$ is similar for $M \|[100]$ and $M \|[111]$.

\section{E. Expansion in directional cosines}

The expansion of the right side of Eq. (10) in a polynomial of the $\alpha_{i}$ 's leads to the following expression for the noncubic charge distribution:

$$
\left\langle l_{z^{\prime}}^{2}-\frac{l(l+1)}{3}\right\rangle=\sum_{i j m n} d_{i j m n} \alpha_{i} \alpha_{j} \alpha_{m} \alpha_{n}+\sum_{i j} d_{i j}^{\prime} \alpha_{i} \alpha_{j} .
$$

The noncubic charge distribution is thus a polynomial of fourth order in the directional cosines of the magnetization. All details of the matrix elements and the band structure are put into the coefficients $d_{i j m n}$ and $d_{i j}^{\prime}$. The important point is again that these coefficients obey the same symmetry as the system without SOC. This allows a particularly transparent formulation of some symmetry properties.

Equation (34) is derived in the same way as its counterpart for the orbital moment, Eq. (23). The only complication is that there are now not only terms like Eqs. (30) and (31) that arise from the longitudinal component of the SOC but also terms like Eqs. (32) and (33) that arise from the transverse component of the SOC. The longitudinal component is proportional to $\sum_{i} \alpha_{i} l_{i}$ and thus automatically leads to a polynomial in the $\alpha_{i}$ 's. However, it can be shown that the terms from the transverse component can also be brought into the form of a polynomial in the $\alpha_{i}^{\prime}$ 's.

The form of the anisotropy directly follows from Eq. (34), since any fourth-order polynomial with cubic symmetric coefficients must have the form

$$
d^{(0)}+d^{(2)}\left(\alpha_{x}^{2} \alpha_{y}^{2}+\alpha_{y}^{2} \alpha_{z}^{2}+\alpha_{z}^{2} \alpha_{x}^{2}\right) .
$$

This form of the anisotropy is thus the consequence of the fourfold appearance of the orbital moment operator and of the cubic lattice symmetry.

The order of the effect can also be deduced directly from Eq. (34): Only the coefficients of even-order terms do not vanish in an expansion like Eq. (34) because of the inversion symmetry of the system. This has the consequence that the lowest-order term is of second order. The next-higher-order terms are of fourth, sixth, ... order and are proportional to $(\xi / W)^{4},(\xi / W)^{6}, \ldots$.

\section{F. Simplified model}

The orbital moment is expressed in Eq. (26) by $N_{o}^{\uparrow}, N_{o}^{\downarrow}$, $\xi$, and $W$. We have tried to derive a similarly simple expression for the noncubic charge distribution. No simple relationship was found, however, which takes the strong cancellation between the $\uparrow \uparrow \uparrow, \uparrow \downarrow \uparrow, \downarrow \uparrow \downarrow$, and $\downarrow \downarrow \downarrow$ contributions adequately into account. Therefore, we derive here only an estimate for the $\uparrow \uparrow \uparrow$ contribution.

The energy splittings in Eq. (28) are replaced by an "appropriate" average $\Delta e$. All details of the density of states are neglected in this way. $1 /(\Delta e)^{2}$ is approximated by

$$
(2 / W)^{3} \int_{0}^{W / 2} d e \int_{W / 2}^{W} d e^{\prime} \int_{W / 2}^{W} d e^{\prime \prime} \frac{1}{\left(e-e^{\prime}\right)\left(e-e^{\prime \prime}\right)} .
$$

This gives $1 /(\Delta e)^{2} \approx 10.5 / W^{2}$. Using this estimate and Eqs. (24) and (25), we obtain from Eq. (28)

$$
\left\langle l_{z^{\prime}}^{2}-\frac{l(l+1)}{3}\right\rangle^{\uparrow \uparrow \uparrow} \approx 0.88(\xi / W)^{2} N_{o}^{\uparrow} N_{u}^{\uparrow}\left(N_{u}^{\uparrow}-N_{o}^{\uparrow}\right) .
$$

The relation (35) is used here to estimate the typical magnitude of the effect. The maximum and minimum of the product $N_{o} N_{u}\left(N_{o}-N_{u}\right)$ are +12.0 and -12.0 . The noncubic charge distribution should, therefore, range in principle between about +10 and $-10(\xi / W)^{2}$. This is in accordance with the model calculations in Figs. 4 and 5, for example. Of course, other factors also play a role. The strong cancellation between the $\uparrow \uparrow \uparrow, \uparrow \downarrow \uparrow, ~ \downarrow \uparrow \downarrow$, and $\downarrow \downarrow \downarrow$ contributions, for example, tends to reduce the effect. On the other hand, peaks in the density of states will enhance the effect, since the effective bandwidth becomes smaller near the peaks.

\section{V. $5 d$ IMPURITIES IN Fe}

\section{A. Local band structure}

Various ab initio calculations of the electronic structure of the $5 d$ impurities in Fe have been performed in the past to investigate the local moments, the orbital moments, the hyperfine fields, and the nuclear spin-lattice relaxation. ${ }^{17,29}$ 


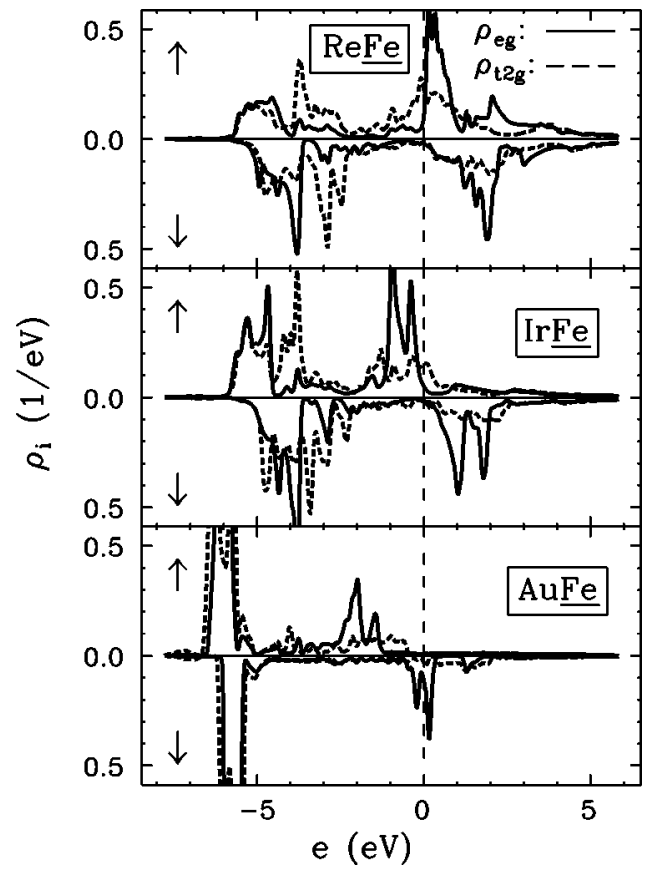

FIG. 6. Partial densities of states for $\operatorname{Re} F e, \operatorname{Ir} F e$, and $\mathrm{Au} F e$.

However, no calculation of the SO-EFG's has been reported. In order to have the densities of states necessary for the tight-binding analysis outlined above, we have calculated the local band structure of the $5 d$ impurities in Fe from Ta to $\mathrm{Au}$ within the full-potential linearized augmented plane-wave (FPLAPW) method using the wien97 code. $^{30}$

The impurity problem was approximated by constructing a supercell with seven $\mathrm{Fe}$ and one impurity atom ( $X \mathrm{Fe}_{7}$ cell). This rather small supercell was chosen to reduce the numerical effort. It should allow us to reproduce the main features in the systematics, but it presents certainly a severe approximation for quantitative purposes. Therefore, the respective set of local band structures should be viewed only as a realistic model for the actual band structures of the $5 d$ impurities in Fe.

Relativistic effects were taken into account within the scalar relativistic approximation. The density functional with generalized-gradient corrections from Ref. 31 was used. The radius $R_{\mathrm{MT}}$ of the atomic spheres was relatively large: $R_{\mathrm{MT}}$ $=2.2$ a.u. for $\mathrm{Fe}$ and $R_{\mathrm{MT}}=2.4$ a.u. for the impurity. All calculations were performed with $k_{\max } R_{\mathrm{MT}}=8$ and a rather coarse mesh of $20 k$ points in the irreducible wedge of the Brillouin zone. The unit cell dimensions were determined self-consistently from the calculated total energies. Thus the effect of the lattice relaxation around the impurity could be approximately incorporated.

The $e_{g}$ and $t_{2 g}$ densities were extracted from the wave functions within the impurity sphere. Since the $d$ wave functions extend beyond $R_{\mathrm{MT}}$, they were normalized to two $e_{g}$ and three $t_{2 g}$ states per impurity and spin direction in the total $d$ band. This procedure is somewhat arbitrary since the upper end of the $d$ band is not uniquely defined.

Figure 6 illustrates the variation of the band structure with the impurity. It shows the $e_{g}$ and $t_{2 g}$ densities of states for $\mathrm{Re} F e, \operatorname{Ir} F e$, and $\mathrm{AuFe}$. The local $d$ band has a characteris- tic basic pattern that remains preserved throughout the $5 d$ series: The densities of states concentrate at both ends of the band. This leads to a distinct separation of the band into an upper and a lower part. The spin-down band lies always above the spin-up band. This is remarkable since the sign of the local moment changes from negative for Re to positive for Ir and Au.

This pattern arises from the adjustment to the host band structure and was already found in a similar form for the $4 d$ impurities in Co and Ni (Refs. 32 and 33): The $4 d$ and $5 d$ orbitals are more extended than the $3 d$ orbitals of the host. Therefore, the bonding and antibonding states tend to be shifted out of the host band and concentrate at both ends of the band. Furthermore, the exchange integral is smaller at the $4 d$ and $5 d$ impurities than in the host. Therefore, the local magnetism is suppressed at the impurity and the relative position of the spin up and the spin down band is mainly determined by the host band structure.

A common basic pattern is also found for the difference between the $e_{g}$ and $t_{2 g}$ densities of states: The $e_{g}$ states are concentrated in the upper part of the band in one prominent density of states peak, whereas the $t_{2 g}$ density is distinctly less peaked in this energy region. This feature is already present in the pure Fe host [see Fig. 2(c)].

But there are also some distinct changes in the local band structure as the atomic number of the impurity increases. The main trends are the following: (i) The center of the band shifts downwards or, in other words, the Fermi energy moves upwards within the common band structure pattern. This band filling is, however, only in part responsible for the increase in the electron number. (ii) The lower part of the $d$ band becomes narrower and its amplitude increases at the expense of the amplitude of the upper part. An important part of the increase in the electron number is due to this effect. (iii) For the light $5 d$ impurities up to Re the amplitude of the lower part of the $\uparrow$ band is smaller than the amplitude of the corresponding part of the $\downarrow$ band. This effect is responsible for the negative local moment in these systems.

\section{B. Orbital moment}

To demonstrate that our calculations give realistic results with regard to the magnetism of the investigated systems, the calculated local spin and orbital moments are compared in Fig. 7 with previous results from circular magnetic x-ray dicroism (CMXD) measurements and fully relativistic spinpolarized Korringa-Kohn-Rostocker (SP-KKR) calculations. ${ }^{34,35}$ The systematic trend that was found in the previous studies is approximately reproduced by our calculations.

The FPLAPW moments in Fig. 7 are the spin and orbital moments of the $d$ electrons in the impurity sphere, however, in order to take the extension of the wave functions beyond $R_{\mathrm{MT}}$ into account, multiplied by the same factor by which the number of $d$ states (occupied and unoccupied) in the sphere is reduced with respect to the nominal number of 10 . To obtain the orbital moment, the SOC was introduced in a second variational $\operatorname{step}^{36}$ as a potential of the form 


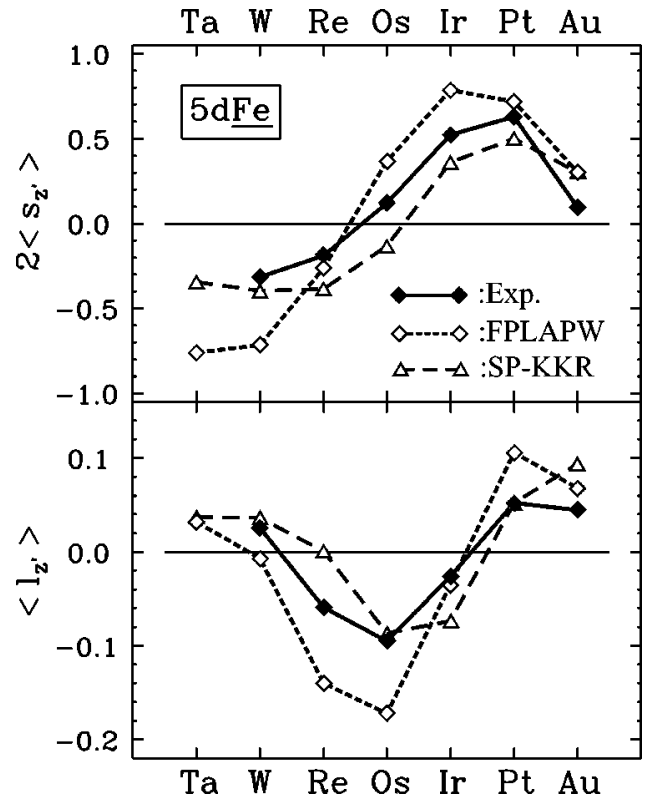

FIG. 7. Spin and orbital moment of the $5 d$ impurities in Fe. The results of our FPLAPW calculations are compared with the results from CMXD measurements (Exp.) and fully relativistic SP-KKR calculations. The CMXD and SP-KKR results are taken from Ref. 35.

$$
\frac{1}{2}\left(\frac{\hbar}{m c}\right)^{2} \frac{1}{r} \frac{\partial V}{\partial r}
$$

within the atomic spheres. The FPLAPW calculation was brought to convergence with this additional potential, and $\left\langle l_{z^{\prime}}\right\rangle$ was extracted from the wave functions within the sphere.

The deviations between the curves in Fig. 7 are at present difficult to interpret: The small supercell used in our calculations is certainly a severe approximation. In the SPR-KKR work the modification of the electronic structure on the neighboring host atoms was not taken into account. Finally, the CMXD results were obtained by an unjustified version of the CMXD sum rules. Therefore, a thorough reexamination of the magnetism of these systems seems to be worthwhile. Moreover, the average moment in the Wigner-Seitz sphere, which was calculated in the SP-KKR calculations, will in general not be identical to the average moment in the impurity sphere of the FPLAPW calculation or the average moment in the vicinity of the nucleus, which is probed by the CMXD measurements.

To investigate the origin of the systematic trend of the orbital moment, the tight-binding analysis was applied. In the upper part of Fig. 8 the orbital moments that were obtained by the application of Eq. (11) to the densities of states are compared with the orbital moments from the FPLAPW calculation. $\xi$ was taken from Ref. 28. Since both approaches are based on the same band structure, the deviations between the two calculations can be directly attributed to the simplified treatment of the SOC and the band structure in the tightbinding analysis. It turns out that not only the systematic trend but also the magnitude of the effect is rather well re-

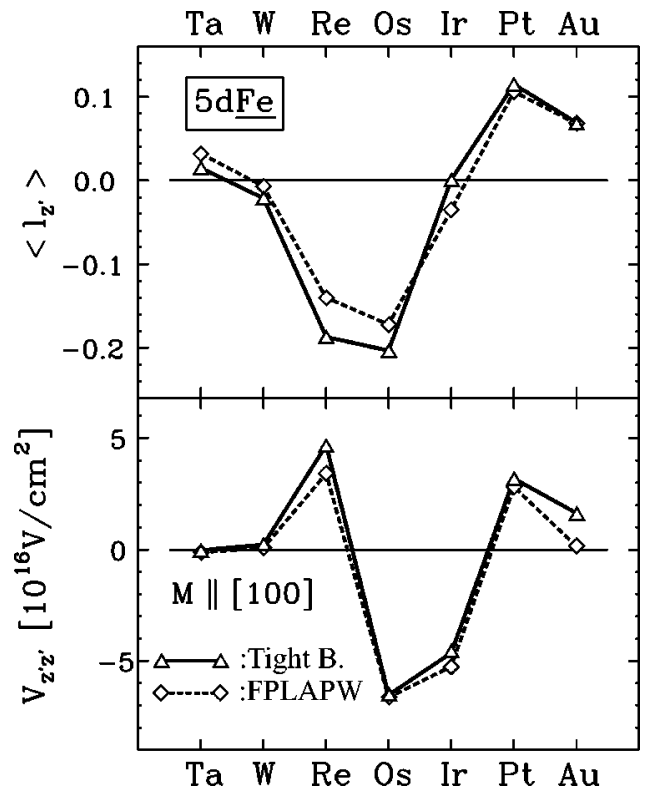

FIG. 8. Direct comparison between tight-binding analysis and $a b$ initio calculation. The orbital moments (top) and noncubic charge distributions (bottom) of the $5 d$ impurities in Fe were either calculated within the FPLAPW approach (FPLAPW) or the density of states from the FPLAPW calculation was used as input for the tight-binding analysis (Tight B.).

produced by the tight-binding analysis. The latter result is remarkable since we have used, for example, $\xi$ 's that were calculated for the free atom.

Figure 9 shows the orbital moment as a function of the band filling for $\operatorname{Re} F e, \operatorname{IrFe}$, and $\mathrm{AuFe}$. To generalize the discussion, the orbital moment is given in units of $\xi / W$, where $W=5.5 \mathrm{eV}$ is the bandwidth of the Fe host.

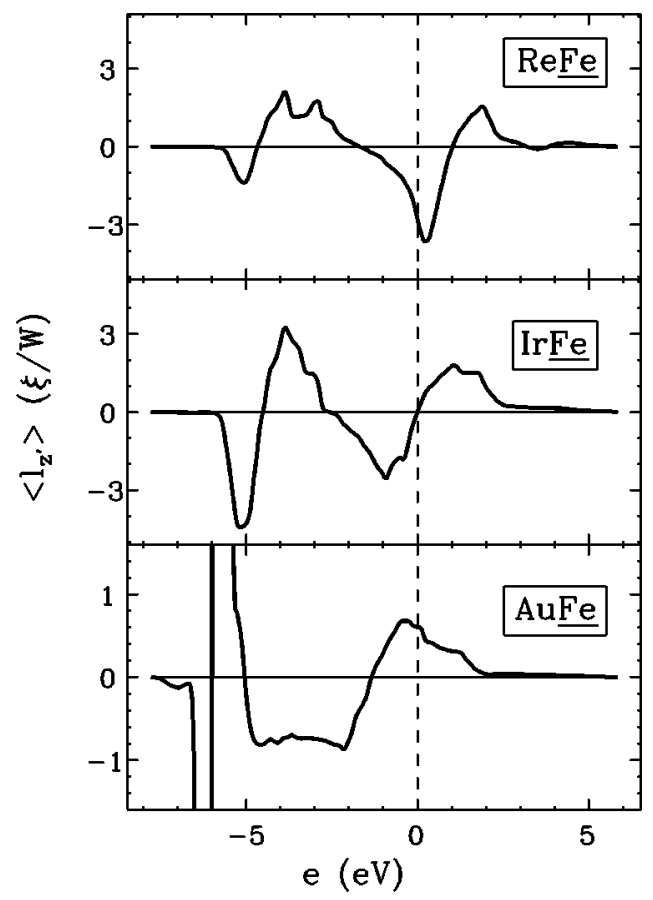

FIG. 9. Orbital moment as a function of the Fermi energy for $\mathrm{Re} F e, \operatorname{IrFe}$, and $\mathrm{AuFe}$. 


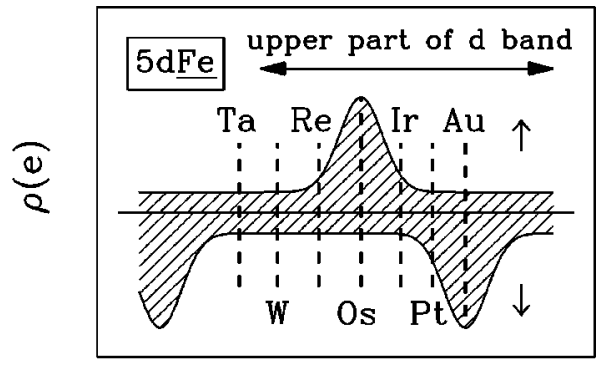

e

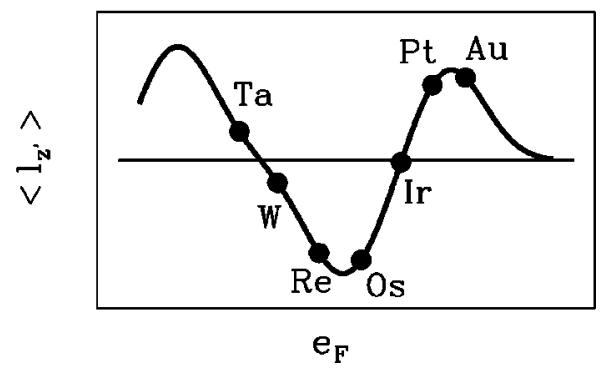

FIG. 10. $5 d$ impurities in Fe: schematic view of the common pattern in the density of states (top) and the orbital moment as a function of $e_{F}$ (bottom). The lower part of the band is largely omitted. The position of $e_{F}$ for the various $5 d$ impurities is marked by the dashed lines and solid circles.

Figure 9 reveals that the variation of the orbital moment with the band filling also shows a common pattern throughout the $5 d$ series. It can be traced back to the common pattern in the band structure: Figure 3(a) has shown the orbital moment as a function of $e_{F}$ for a simple ferromagnetic band structure. Due to the separation of the $5 d$ band into two parts, this function is now found for each of the two parts and thus appears 2 times in series. With increasing atomic number the Fermi energy moves successively upwards within this pattern, in accordance with the respective upward move within the common band structure pattern. The general trend of the systematics can thus be described as a band filling effect. The orientation of the local spin moment seems to play only a minor role.

This interpretation of the systematics of the orbital moment as a band filling effect is schematically shown in Fig. 10. No scales are given on the axes since only the basic pattern of the dependence on $e_{F}$ remains preserved throughout the $5 d$ series. The widths and amplitudes of the various parts of this dependence vary considerably. Comparison of Figs. 6 and 9 shows that in this respect similar trends can be observed for the density of states and orbital moment.

\section{Noncubic charge distribution}

To calculate the SO-EFG within the FPLAPW method, the SOC was introduced as an additional potential as in the case of the orbital moment, and the expectation value $\left\langle\left(1 / r^{3}\right)\left[l_{z^{\prime}}^{2}-l(l+1) / 3\right]\right\rangle$ was evaluated for the $d$ electrons within the atomic spheres. Alternatively, the noncubic charge distribution was calculated by applying Eq. (15) to the densities of states obtained without SOC, and $\xi$ and $\left\langle r^{-3}\right\rangle$ were taken from Ref. 28.

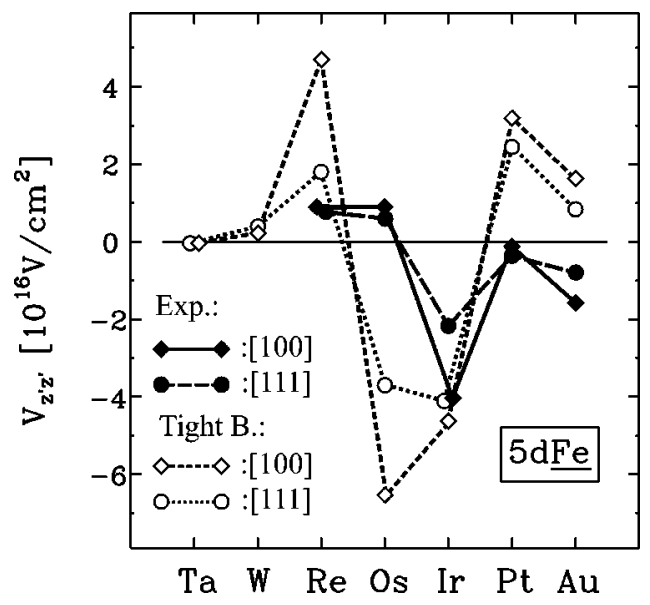

FIG. 11. Noncubic charge distribution of the $5 d$ impurities in $\mathrm{Fe}$. The results of our model calculations (open symbols) are compared with the experiment (solid symbols).

An interesting detail of the FPLAPW calculations was that the SO-EFG's after the convergence of the calculation with SOC were between $17 \%$ and $25 \%$ smaller than the SOEFG's that were obtained by adding the SOC only in the final calculation of the wave functions without further iteration steps. In contrast, the convergence of the band structure after the introduction of the SOC had almost no influence on the orbital moment. This shows that the SOC leaves the band structure essentially unchanged and the observed reduction of the SO-EFG can be interpreted as a shielding effect within the valence electron shell that corresponds to the concept of the atomic Sternheimer shielding factor $R .^{37}$

The SO-EFG's of the FPLAPW calculations and the tightbinding analysis are compared in the bottom part of Fig. 8 . Again, the tight-binding analysis reproduces not only the systematic trend but also the magnitude of the effect remarkably well, despite the various approximations such as the perturbation treatment of the SOC, the use of densities of states instead of the complete band structure, the use of atomic constants for $\xi$ and $\left\langle r^{-3}\right\rangle$, and the neglect of the shielding effect.

Since FPLAPW calculations were performed only for $M \|[100]$, we compare in Fig. 11 the experimental numbers with the results of the tight-binding analysis. The comparison reveals similarities in the overall behavior of the effect - for example, in the strong variation of the SO-EFG with the impurity or the tendency for the [100] SO-EFG to be larger than the [111] SO-EFG - but also large deviations for the individual systems. We interpret this in the following way: The calculated band structures are not accurate enough to reproduce the SO-EFG for a given impurity. The better agreement with the experiment in the case of the orbital moment is due to the smaller sensitivity of that quantity to band structure details. However, the calculations should be realistic enough to correctly reproduce the basic relations between the noncubic charge distribution and the band structure. Why the calculations overestimate the magnitude of the effect is at present not clear.

To obtain insight into the origin of the main systematic trends, the dependence on the band filling was studied in 


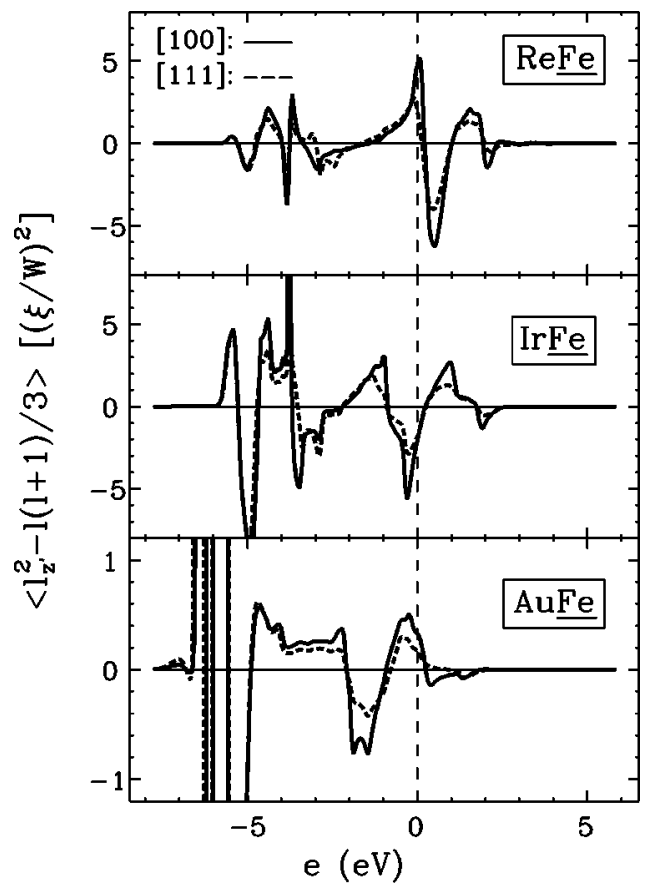

FIG. 12. Noncubic charge distribution as a function of the Fermi energy for $\mathrm{Re} F e, \operatorname{IrFe}$, and $\mathrm{AuFe}$.

more detail. Figure 12 shows the dependence of the noncubic charge distribution on the band filling for $\operatorname{Re} F e, \operatorname{Ir} F e$, and $\mathrm{AuFe} .\left\langle l_{z^{\prime}}^{2}-l(l+1) / 3\right\rangle$ is given in units of $(\xi / W)^{2}$. In all cases $W=5.5 \mathrm{eV}$ was assumed.

Again, we find a common pattern in the dependence on the band filling and an upward move of the Fermi energy within this pattern: The noncubic charge distribution as a function of $e_{F}$ has been shown in Fig. 5(a) for a simple ferromagnetic band structure. Due to the separation of the $5 d$ band into two parts, this function is now found for each of the two parts and thus appears 2 times in series. The position of the Fermi energy within this pattern moves upwards with increasing atomic number, in accordance with the respective upward move of $e_{F}$ within the common band structure pattern. Figure 13 schematically shows the calculated positions of the Fermi energy within this band filling scheme for the various $5 d$ impurities in Fe. Since the calculations fail to reproduce the experimental SO-EFG's, the actual positions of the Fermi energy within the scheme must be different from the ones shown in Fig. 13. However, the scheme itself should be essentially correct.

There is also a common trend in the anisotropy: The [100] noncubic charge distribution is usually larger than the [111] noncubic charge distribution, although the form of the dependence on $e_{F}$ is rather similar for $M \|[100]$ and $M \|[111]$. We found this effect already in Sec. IV D for pure Fe and explained it as a consequence of the concentration of the $e_{g}$ states in the upper part of the $d$ band into one strong density of states peak. This concentration is also found for all $5 d$ impurities and seems thus to be a property of the bcc lattice.

The band filling scheme in Fig. 13 explains the gross features of the systematics. But other factors are also important for the actual magnitude of the effect: Figure 12 shows, for example, that it is only the basic pattern of the depen-
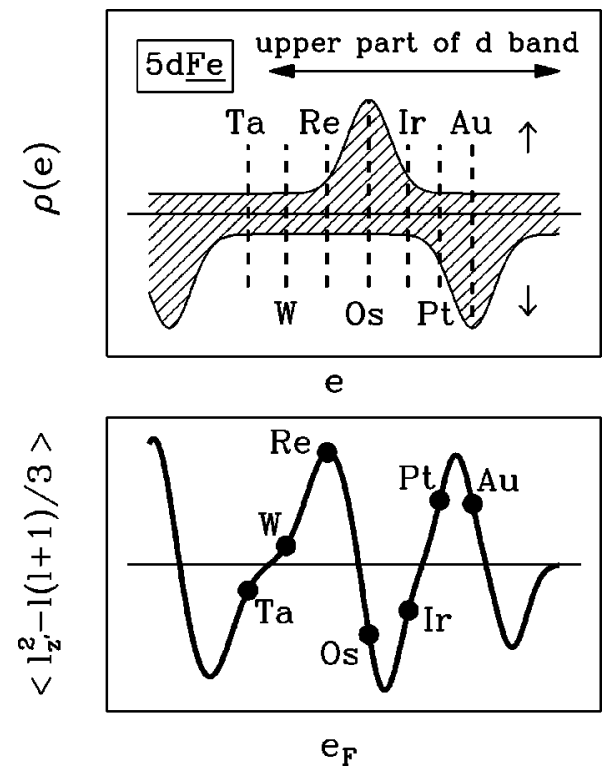

FIG. 13. $5 d$ impurities in Fe: schematic view of the common pattern in the density of states (top) and the noncubic charge distribution as a function of $e_{F}$ (bottom). The positions of $e_{F}$ are the same as in Fig. 10.

dence on $e_{F}$ that remains preserved throughout the $5 d$ series. The widths and amplitudes of the various parts of this dependence vary considerably. The trends are here essentially the same as for the density of states. The reduction of the upper part of the $d$ band with increasing atomic number of the impurity, for example, leads to a rapid decrease of the SO-EFG magnitude for impurities above Ir. The strong increase of the prefactor $\xi^{2}\left\langle 1 / r^{3}\right\rangle$ within the $5 d$ series due to the contraction of the $5 d$ shell is also important. According to Ref. 28, this factor rises from Ta to Au from 0.33 to 5.3 $(\mathrm{eV})^{2} /\left(a_{B}\right)^{3}$. This contributes to the marked decrease of the $\mathrm{SO}-\mathrm{EFG}$ for the light $5 d$ impurities below Re. The fine structure in the dependence on $e_{F}$ that is introduced by the sensitivity to band structure details may also play a role for some systems.

\section{SUMMARY}

The band structure plays a decisive role for the spin-orbit induced noncubic charge distribution. Therefore, it would be desirable to understand this role in more detail. However, the sophisticated treatment of the band structure and the SOC within $a b$ initio calculations, although necessary for the quantitative description, is in general too involved to allow a qualitative understanding. We propose in this work a more transparent but still realistic treatment of the SOC within the tight-binding approximation.

The comparison with more quantitative treatments shows that the proposed tight-binding analysis is realistic enough to reproduce at least qualitatively the systematics. On the other hand, the structure of the resulting equations proves to be transparent enough to explain the basic properties of the effect in terms of the interaction between the SOC and band structure.

Even if the band structure deviates significantly from a 
simple exchange-split ferromagnet, the main features of the orbital moment and the noncubic charge distribution may be obtained by the analysis of the band structure calculated without SOC. This was demonstrated for the $5 d$ impurities in $\mathrm{Fe}$.

\section{APPENDIX A: RIGID SHIFT MODEL}

The SOC was treated in the first papers on the SO-EFG within a model that will be referred to in the following as the rigid shift (RS) model. It was so far the only available model for the SO-EFG and it is attractively simple. The more complete tight-binding analysis allows us now to investigate whether the RS model is able to reproduce the essential physics.

For a detailed derivation and discussion of the RS model we refer to the original papers. ${ }^{14-16}$ We will repeat here only the main points. The basic idea is that the partial density of states of the orbital with orbital and spin magnetic quantum numbers $m_{l}$ and $m_{s}$ is rigidly shifted by the SOC by $\xi m_{l} m_{s}$. Assuming that in the absence of the SOC the partial densities of states are independent of $m_{l}$, the following expressions for the orbital moment and the noncubic charge distribution can be derived in the limit of small $\xi$ 's (Ref. 16):

$$
\begin{gathered}
\left\langle l_{z^{\prime}}\right\rangle=\xi\left[\rho^{\downarrow}\left(e_{F}\right)-\rho^{\uparrow}\left(e_{F}\right)\right], \\
\left\langle l_{z^{\prime}}^{2}-\frac{l(l+1)}{3}\right\rangle=\xi^{2}(7 / 20)\left[\rho^{\prime \uparrow}\left(e_{F}\right)+\rho^{\prime} \downarrow\left(e_{F}\right)\right] .
\end{gathered}
$$

$\rho^{\uparrow}(e)$ is here the total density of $\uparrow$ states and $\rho^{\prime \uparrow}(e)$ $=d \rho^{\uparrow}(e) / d e$ its derivative.

This model was extended by the inclusion of the crystal potential that splits the energy of the $e_{g}$ and $t_{2 g}$ orbitals. ${ }^{15,16}$ In the extended version the ten $d$ orbitals are first diagonalized with respect to the exchange splitting, the crystal potential, and the SOC. Then, in the spirit of the rigid shift of the densities of states, the partial densities of states are assumed to have all the same shape and are centered on the respective eigenenergies.

The main difference to the tight-binding analysis is that the SOC shifts the energy of the states instead of mixing the states. This makes the final equations much simpler. But it ignores an essential part of the physics, since in reality the states are mixed rather than shifted in energy. This leads to several even qualitatively wrong predictions that demonstrate the inadequacy of the RS model.

(i) According to the RS model, only the density of states at the Fermi energy is important. Since the density of states is a strongly varying function, this would lead to a large sensitivity to band structure details. Actually, however, the SOC mixes states from above and below the Fermi energy. Therefore, the whole band is important, and the sensitivity to band structure details is much weaker than implied by Eqs. (A1) and (A2). A prominent example is the orbital moment of Fe: Due to the marked dip in the spin-down density of states just at the Fermi energy [see Fig. 2(c)], Eq. (A1) predicts a negative orbital moment. But in accordance with Eq. (26), the orbital moment of $\mathrm{Fe}$ is positive. (ii) In the RS model, the mixing of $\uparrow$ and $\downarrow$ states by the SOC is strongly suppressed by their exchange splitting, which is usually much larger than the SOC. The $\uparrow \downarrow \uparrow$ and $\downarrow \uparrow \downarrow$ contributions to the noncubic charge distribution, for example, should accordingly be of minor importance. The fault of the RS model is here that the broadening of the states into bands is completely ignored when the mixing of the states by the SOC is considered. Actually, the large overlap between $\uparrow$ and $\downarrow$ bands leads to an equally strong mixing between the bands as within the bands. The $\uparrow \downarrow \uparrow$ and $\downarrow \uparrow \downarrow$ contributions are, therefore, as important as the $\uparrow \uparrow \uparrow$ and $\downarrow \downarrow \downarrow$ contributions.

(iii) In the RS model, the partial $e_{g}$ and $t_{2 g}$ densities of states are simply shifted with respect to each other by the crystal potential. But in reality, the $e_{g}$ and $t_{2 g}$ bands can differ in many other ways. The RS model is, therefore, in general inadequate to describe the anisotropy of the noncubic charge distribution for realistic systems.

(iv) The anisotropy of the noncubic charge distribution arises in the RS model from the suppression of the mixing of $t_{2 g}$ and $e_{g}$ states by the crystal field splitting $V_{c}$ of these states. Accordingly, it should decisively depend on the ratio $V_{c} / \xi$. Again, the fault of the RS model is that the broadening of the states into bands is completely ignored for the mixing of the states. Actually, the $t_{2 g}$ and $e_{g}$ bands largely overlap. The mixing between $t_{2 g}$ and $e_{g}$ states is, therefore, even for arbitrarily small $\xi$ 's, about as strong as the mixing between the $t_{2 g}$ states, and the anisotropy depends primarily only on the different form of the $e_{g}$ and $t_{2 g}$ bands and not on $\xi$.

\section{APPENDIX B: LOCALIZED AND HOMOGENEOUS SPIN-ORBIT COUPLING}

We have assumed in Sec. II that the SOC is localized at the site of the impurity. This should be a good approximation for heavy impurities where the SOC is much larger than in the host. To see what happens if the SOC at the impurity and in the host are of the same order of magnitude, we will now investigate the opposite extreme, the pure ferromagnetic metal, where the SOC strength is the same on all lattice sites. First-order nondegenerate perturbation theory gives in this case the following expression for the orbital moment ${ }^{27}$ :

$$
\begin{aligned}
\left\langle l_{z^{\prime}}\right\rangle= & \int \frac{d^{3} k}{V_{B}} \sum_{\substack{i \\
e_{i \vec{k}}<e_{F}}} \sum_{\substack{j \\
e_{j}>e_{F}}} \frac{\left\langle\phi_{i \vec{k}}\left|l_{z^{\prime}}\right| \phi_{j \vec{k}}\right\rangle\left\langle\phi_{j \vec{k}}|\vec{\xi} \cdot \vec{l}| \phi_{i \vec{k}}\right\rangle}{e_{i \vec{k}}-e_{j \vec{k}}} \\
& +\frac{\left\langle\phi_{i \vec{k}}|\xi \vec{s} \cdot \vec{l}| \phi_{j \vec{k}}\right\rangle\left\langle\phi_{j \vec{k}}\left|l_{z^{\prime}}\right| \phi_{i \vec{k}}\right\rangle}{e_{i \vec{k}}-e_{j \vec{k}}} .
\end{aligned}
$$

$\phi_{i \vec{k}}$ and $e_{i \vec{k}}$ are the tight-binding orbitals and the energies of the eigenstates in the absence of the SOC. $\vec{k}$ is the wave vector, $i$ the band index. $V_{B}$ is the volume of the Brillouin zone. We neglect here points in the Brillouin zone with high symmetry, where in general some $e_{i \vec{k}}$ s are degenerate and nondegenerate perturbation theory is not applicable.

The orbital moment at a heavy impurity can be expressed in a similar form: If the Green's functions in Eq. (9) are expressed in terms of the eigenstates by using Eqs. (2) and (4), we obtain 


$$
\left\langle l_{z^{\prime}}\right\rangle=\int \frac{d^{3} k}{V_{B}} \sum_{\substack{i \\ e_{i \vec{k}}<e_{F}}} \int \frac{d^{3} k^{\prime}}{V_{B}} \sum_{\substack{j \\ e_{j k^{\prime}}>e_{F}}} \frac{\left\langle\phi_{i \vec{k}}\left|l_{z^{\prime}}\right| \phi_{j \overrightarrow{k^{\prime}}}\right\rangle\left\langle\phi_{j \vec{k}^{\prime}}|\xi \vec{\xi} \cdot \vec{l}| \phi_{i \vec{k}}\right\rangle}{e_{i \vec{k}}-e_{j \vec{k}^{\prime}}}+\frac{\left\langle\phi_{i \vec{k}}|\xi \vec{s} \cdot \vec{l}| \phi_{j \overrightarrow{k^{\prime}}}\right\rangle\left\langle\phi_{j \vec{k}^{\prime}}\left|l_{z^{\prime}}\right| \phi_{i \vec{k}}\right\rangle}{e_{i \vec{k}}-e_{j \vec{k}^{\prime}}}
$$

We conclude that the equations for the pure metal (homogeneous SOC) and for a heavy impurity (localized SOC) have essentially the same structure. The difference is that in the pure metal only states with the same wave vector are mixed, whereas the localized SOC mixes all states irrespective of their wave vectors. If in Eq. (B1) $\left|\phi_{j k}\right\rangle\left\langle\phi_{j \vec{k}}\right|$ is replaced by its average over all $\phi_{j \vec{k}}$ 's of the same energy, one obtains Eq. (B2).

The use of Eq. (11) for the pure metal is thus equivalent to the averaging over all states of the same energy and the neglect of the symmetry points in the Brillouin zone. Only explicit calculations can clarify to which extent this is a good approximation. But from the similar structure of the equations one can expect that most of the conclusions in this work still hold even if the SOC's at the impurity and host are of the same order of magnitude.

In any case, the averaging over all states of the same energy is a necessary simplification to obtain a transparent model, since it allows us to combine all states of the same energy into the density of states. It would be impossible to keep the overview over the complete set of eigenstates and eigenenergies. Thus, Eq. (11) will perhaps work for the $3 d$ and $4 d$ impurities not as well as for the $5 d$ impurities, but it is the best we can do if we do not want to make use of the full band structure.

Similar conclusions can also be drawn for the noncubic charge distribution.

\section{APPENDIX C: NONCUBIC CHARGE DISTRIBUTION AND MAGNETOSTRICTION}

There is no doubt that the magnetostriction contributes to the noncubic charge distribution and the noncubic charge distribution to the magnetostriction. In transition metals, however, for both effects other mechanisms are thought to be more important. (For the rare earths the noncubic $f$ shell is indeed thought to be the main source of the magnetostriction. ${ }^{38}$ ) In the absence of ab initio calculations we have to restrict ourselves to order of magnitude estimates.

The magnetostriction causes in $\mathrm{Fe}, \mathrm{Co}$, and $\mathrm{Ni}$ relative length changes $d l / l$ along the direction of the magnetization of the order of $5 \times 10^{-5} .39$ To get an idea of the EFG strengths that we have to expect from such a small lattice distortion we refer to the EFG's in hexagonal metals. The typical EFG strength per $10^{-2}$ deviation of the $c / a$ ratio from the ideal ratio 1.633 is of the order of $2 \times 10^{16} \mathrm{~V} / \mathrm{cm}^{2}$ for $3 d$ and $4 d$ elements and of the order of $2 \times 10^{17} \mathrm{~V} / \mathrm{cm}^{2}$ for $5 d$ elements. ${ }^{40}$ If we take into account that, at least in a point charge model, $V_{z z} /(d l / l)$ in a cubic metal is only about a third of $V_{z z} /(c / a-1.633)$ in hexagonal metals, ${ }^{41}$ we ex- pect magnetostriction-induced EFG's of the order of 3 $\times 10^{13} \mathrm{~V} / \mathrm{cm}^{2}$ for the $3 d$ and $4 d$ impurities and of the order of $3 \times 10^{14} \mathrm{~V} / \mathrm{cm}^{2}$ for the $5 d$ impurities. In contrast, the respective SO-EFG's are of the order of $10^{15} \mathrm{~V} / \mathrm{cm}^{2}$ and $10^{16} \mathrm{~V} / \mathrm{cm}^{2}{ }^{7}$ Thus, the magnetostriction seems to be not an important contribution to the noncubic charge distribution. This conclusion was already drawn in Ref. 15.

The noncubic charge distribution represents also a quadrupole moment $Q$ of the valence electron shell. Its energy in the presence of an EFG $V$ is given by

$$
E_{q}=(1 / 4) Q_{z^{\prime} z^{\prime}} V_{z^{\prime} z^{\prime}},
$$

where we have assumed axial symmetry along the direction $z^{\prime}$ of the magnetization. There is no EFG in cubic lattice symmetry, but due to $E_{q}$, the energy of the system would be lowered if there is one. This will distort the lattice until the gain in $E_{q}$, which is linear in the distortion, is compensated for by the loss in elastic energy, which is quadratic in the distortion.

We assume in Eq. (C1) a pure electrostatic interaction between the noncubic charge distribution and the lattice distortion because we want to obtain in a simple way an order of magnitude estimate. Of course, the real nature of this interaction is more complex. For simplicity, we assume also that $Q, V$, the lattice distortion, and the magnetoelastic coupling constant are isotropic and axially symmetric with respect to the direction of the magnetization.

The quadrupole moment of the $d$ shell can be estimated as

$$
Q_{z^{\prime} z^{\prime}}=(1 / 3) e\left\langle r^{2}\right\rangle\left\langle l_{z^{\prime}}^{2}-\frac{l(l+1)}{3}\right\rangle
$$

The EFG connected with a relative length change $d l / l$ is estimated within a point charge model by ${ }^{41}$

$$
V_{z^{\prime} z^{\prime}} \approx-1.5 \frac{Z e}{a^{3}}(d l / l)
$$

$Z$ is the effective point charge and $a$ the cubic lattice constant. Combining Eqs. (C1) and (C3) we obtain a magnetoelastic energy of the form $E_{q}=B(d l / l)$. Assuming $Z=1, a$ $=2.86 \AA$, a spin-orbit induced $\left\langle l_{z^{\prime}}^{2}-l(l+1) / 3\right\rangle$ of 0.9 $\times 10^{-3},{ }^{7}$ and $\left\langle r^{2}\right\rangle=0.5 \AA^{2},{ }^{28}$ we obtain for the magnetoelastic coupling constant $B=-0.030 \mathrm{meV} / \mathrm{atom}$.

The actual magnetoelastic coupling constant for $\mathrm{Fe}$, $M \|[100]$, is $B_{1}=-0.25 \mathrm{meV} / \mathrm{atom},{ }^{39}$ an order of magnitude larger. Thus, the noncubic charge distribution seems to be not the dominant source of the magnetostriction. However, in view of the crude nature of our estimates, this point would deserve a more exact study. 
${ }^{1}$ T. Gasche, M. S. S. Brooks, and B. Johansson, Phys. Rev. B 53, 296 (1996).

${ }^{2}$ J. Trygg, B. Johansson, O. Eriksson, and J. M. Wills, Phys. Rev. Lett. 75, 2871 (1995).

${ }^{3}$ R. Wu, L. Chen, and A. J. Freeman, J. Magn. Magn. Mater. 170, 103 (1997).

${ }^{4}$ W. B. Zeper, F. J. A. M. Greidanus, P. F. Carcia, and C. R. Fincher, J. Appl. Phys. 65, 4971 (1989).

${ }^{5}$ M. T. Johnson, P. J. H. Bloemen, F. J. A. den Broeder, and J. J. de Vries, Rep. Prog. Phys. 59, 1409 (1996).

${ }^{6}$ O. Hjortstam, K. Baberschke, J. M. Wills, B. Johansson, and O. Eriksson, Phys. Rev. B 55, 15026 (1997).

${ }^{7}$ G. Seewald, E. Zech, E. Hagn, R. Kleyna, M. Voß, D. ForkelWirth, A. Burchard, and ISOLDE Collaboration, preceding paper, Phys. Rev. B 66, 174401 (2002).

${ }^{8}$ O. Eriksson, L. Nordström, A. Pohl, L. Severin, A. M. Boring, and B. Johansson, Phys. Rev. B 41, 11807 (1990).

${ }^{9}$ H. Ebert, M. Battocletti, and E. K. U. Gross, Europhys. Lett. 40, 545 (1997).

${ }^{10}$ T. Huhne, C. Zecha, H. Ebert, P. H. Dederichs, and R. Zeller, Phys. Rev. B 58, 10236 (1998).

${ }^{11}$ P. Blaha, K. Schwarz, and P. H. Dederichs, Phys. Rev. B 37, 2792 (1988).

${ }^{12}$ P. Dufek, P. Blaha, and K. Schwarz, Phys. Rev. Lett. 75, 3545 (1995).

${ }^{13}$ H. Haas, Hyperfine Interact. 120/121, 157 (1999).

${ }^{14}$ M. Aiga and J. Itoh, J. Phys. Soc. Jpn. 31, 1844 (1971).

${ }^{15}$ G. A. Gehring and H. C. W. L. Williams, J. Phys. F: Met. Phys. 4, 291 (1974).

${ }^{16}$ M. Aiga and J. Itoh, J. Phys. Soc. Jpn. 37, 967 (1974).

${ }^{17}$ H. Ebert, R. Zeller, B. Drittler, and P. H. Dederichs, J. Appl. Phys. 67, 4576 (1990).

${ }^{18}$ C. Demangeat, J. Phys. F: Met. Phys. 5, 169 (1975).

${ }^{19}$ C. Demangeat, J. Phys. F: Met. Phys. 6, 1637 (1975).

${ }^{20}$ P. A. Wolff, Phys. Rev. 124, 1030 (1961).

${ }^{21}$ T. Moriya, in Theory of Magnetism in Transition Metals, Proceedings of the International School of Physics, Enrico Fermi, Vol. 37, edited by W. Marshal (Academic, New York, 1967), p. 206.
${ }^{22}$ J. C. Slater and G. F. Koster, Phys. Rev. 94, 1498 (1954).

${ }^{23}$ H. Takayama, K.-P. Bohnen, and P. Fulde, Phys. Rev. B 14, 2287 (1976).

${ }^{24}$ M. S. S. Brooks and P. J. Kelly, Phys. Rev. Lett. 51, 1708 (1983).

${ }^{25}$ J. Trygg, B. Johansson, O. Eriksson, and J. M. Wills, Phys. Rev. Lett. 75, 2871 (1995).

${ }^{26}$ H. Brooks, Phys. Rev. 58, 909 (1940).

${ }^{27}$ P. Bruno, Phys. Rev. B 39, 865 (1989); in Ferienkurse des Forschungszentrum Jülich, edited by R. Hölze (Forschungszentrum Jülich, 1993), Chap. 24.

${ }^{28}$ S. Fraga, J. Karwowski, and K. M. S. Saxena, Handbook of Atomic Data (Elsevier, Amsterdam, 1976).

${ }^{29}$ D. H. Akai, Hyperfine Interact. 43, 255 (1988).

${ }^{30}$ P. Blaha, K. Schwarz, and J. Luitz, computer code wien 97, TU Vienna, 1997. An improved and updated Unix version of the original copyrighted WIEN code, published in P. Blaha, K. Schwarz, P. Sorantin, and S. B. Trickey, Comput. Phys. Commun. 59, 399 (1990).

${ }^{31}$ J. P. Perdew, K. Burke, and M. Ernzerhof, Phys. Rev. Lett. 77, 3865 (1996).

${ }^{32}$ R. Zeller, J. Phys. F: Met. Phys. 17, 2123 (1987).

${ }^{33}$ V. S. Stepanyuk, R. Zeller, P. H. Dederichs, and I. Mertig, Phys. Rev. B 49, 5157 (1994).

${ }^{34}$ G. Schütz, M. Knülle, and H. Ebert, Phys. Scr. T49, 302 (1993); G. Schütz and P. Fischer, in Ferienkurse des Forschungszentrum Jülich, edited by R. Hölze (Forschungszentrum, Jülich, 1993), Chap. 22.

${ }^{35}$ H. Ebert, Rep. Prog. Phys. 59, 1665 (1996).

${ }^{36}$ D. J. Singh, Plane Waves, Pseudopotentials and the LAPW Method (Kluwer Academic, Dordrecht, 1994).

${ }^{37}$ E. N. Kaufmann and R. J. Vianden, Rev. Mod. Phys. 51, 161 (1979).

${ }^{38}$ R. Coehoorn, J. Magn. Magn. Mater. 99, 55 (1991).

${ }^{39}$ M. B. Stearns, in Magnetic Properties of Metals, edited by H. P. Wijn, Landolt-Börnstein, New Series, Group III, Vol. 19a (Springer, Berlin, 1986).

${ }^{40}$ R. Vianden, Hyperfine Interact. 15/16, 1081 (1983).

${ }^{41}$ F. W. de Wette, Phys. Rev. 123, 103 (1961). 